\title{
Metabolic factors, lifestyle habits, and possible polyneuropathy in early type 2 diabetes: A nationwide study of 5,249 patients in the Danish DD2 cohort
}

Authors:

Diana H. Christensen, ${ }^{1,2}$ Søren T. Knudsen, ${ }^{3}$ Sandra S. Gylfadottir, ${ }^{2,4,5}$ Lotte B. Christensen, ${ }^{1}$ Jens S. Nielsen, ${ }^{6}$ Henning Beck-Nielsen, ${ }^{6}$ Henrik T. Sørensen, ${ }^{1}$ Henning Andersen, ${ }^{2,5}$ Brian C. Callaghan, ${ }^{2,7}$ Eva L. Feldman, ${ }^{2,7}$ Nanna B. Finnerup, ${ }^{2,4,5}$ Troels S. Jensen, ${ }^{2,4,5}$ Reimar W. Thomsen, ${ }^{1}$

Author affiliations:

${ }^{1}$ Department of Clinical Epidemiology, Institute of Clinical Medicine, Aarhus University Hospital, Aarhus, Denmark

2International Diabetic Neuropathy Consortium, Department of Clinical Medicine, Faculty of Health, Aarhus University, Aarhus, Denmark

${ }^{3}$ Steno Diabetes Center Aarhus (SDCA), Aarhus University Hospital, Aarhus, Denmark

${ }^{4}$ Department of Clinical Medicine, Danish Pain Research Center, Aarhus University, Aarhus, Denmark

${ }^{5}$ Department of Neurology, Aarhus University Hospital, Aarhus, Denmark

${ }^{6}$ Danish Center for Strategic Research in Type 2 Diabetes (DD2), Steno Diabetes Center Odense, Odense, Denmark

${ }^{7}$ Department of Neurology, University of Michigan, Ann Arbor, MI, 48109, USA

Corresponding author:

Diana Christensen, Department of Clinical Epidemiology, Aarhus University Hospital, Olof Palmes Allé 43-45, DK-8200, Aarhus N, Denmark. Tel: +45 8716 8248. Fax: +45 87167215. Email: dhcr@clin.au.dk 


\section{Supplemental material}

The main focus of this study is the association of metabolic and lifestyle factors measured at baseline around time of type 2 diabetes diagnosis with possible DPN and neuropathic pain at a median of 2.8 years later, as well as the association of changes in some factors from age 20 (BMI) or baseline (smoking and physical activity) to possible DPN/pain assessment median 2.8 years after baseline.

Results for the most important baseline risk factors are presented in the table/figures in the main manuscript.

In this supplementary material, we present results for a few additional baseline risk factors; stratified analyses results; analyses of risk factors measured at the time of questionnaire in 2016; additionally adjusted analyses; and analyses of continuous risk factor data. 
Tables:

Page

Supplementary Table 1: Definitions and codes

Supplementary Table 2: Descriptive data

Supplementary Table 3: Obesity measures and association with DPN; results for analyses using continuous data including the analyses using a unit of $1 S D$.

Supplementary Table 4: Sex-stratified analyses: Obesity measures and association with DPN

Supplementary Table 5: Obesity and non-obesity risk factors and the association with DPN; results for analyses using categorical data. This table includes analyses of additional risk factors measured at baseline as well as BMI, smoking, and alcohol (i.e. those factors for which changes are reported) measured at questionnaire time 2016

Supplementary Table 6: Non-obesity risk factors and association with DPN: results for analyses using continuous data

Supplementary Table 7: Obesity and non-obesity risk factors and pain occurrence in DPN; results for analyses using categorical data including analyses of additional risk factors measured at baseline as well as BMI, smoking, and alcohol (i.e. those factors for which changes are reported) measured at questionnaire time 2016

Supplementary Table 8: Obesity measures and pain occurrence in DPN; results for analyses using continuous data including the analyses using a unit of $1 S D$

Supplementary Table 9: Non-obesity measures and association with pain occurrence in DPN; results for analyses using continuous data

Supplementary Table 10: Sex-stratified analyses, Obesity measures and the association with pain occurrence in DPN

Supplementary Table 11: Non-obesity risk factors and the association with DPN: additional adjustment for central obesity and $\mathrm{HbA1C}$

Supplementary Table 12: Obesity measures and the association with DPN: 
Figures:

Page

Supplementary Figure 1: Flowchart 22

Supplementary Figure 2: DPN definitions 23

Supplementary Figure 3: Spline regressions 24

Supplementary Figure 4: Central obesity measures and the association with DPN, 27 additionally adjusted for BMI 


\section{TABLES}

\begin{tabular}{|c|c|}
\hline \multicolumn{2}{|c|}{ Supplementary Table 1: Definitions and codes used in this study } \\
\hline Variable & Definition and codes \\
\hline \multicolumn{2}{|l|}{ DDDA/DD2/IDNC variables } \\
\hline $\begin{array}{l}\text { DDDA variables } \\
\text {-Blood pressure } \\
\text {-Lipids } \\
\text {-HbA1c } \\
\text {-Smoking baseline } \\
\text {-BMI (see anthropometric data) }\end{array}$ & $\begin{array}{l}\text { Categories: } \\
\text { Systolic blood pressure: }</ \geq 130 \\
\text { Diastolic blood pressure: }</ \geq 80 \\
\text { Lipids } \\
\text { LDL: }<1.8 / 1.8-2.6, \geq 2.6 \\
\text { HDL (male/female): }<1.0 / 1.2, \geq 1.0 / 1.2 \\
\text { Triglycerides: }</ \geq 1.7 \\
\text { Total cholesterol: }</ \geq 4.3 \\
\text { HbA1c, mmol/mol: }<48,48-57,58-67,68-77, \geq 78 \\
\text { HbA1c, \%: }<6.5,6.5-7.4,7.5-8.4,8.5-9.4, \geq 9.5 \\
\text { Smoking: Never, former, current (daily + occasionally) } \\
\text { References: } \\
\text { https://www.nbv.cardio.dk/dyslipidaemi } \\
\text { American Diabetes Association } 2003 \\
\text { BMI: see below }\end{array}$ \\
\hline $\begin{array}{l}\text { DD2 core variables } \\
\text {-low-grade inflammation/hsCRP } \\
\text {-C-peptide } \\
\text {-Physical activity, baseline } \\
\text {-Alcohol, baseline } \\
\text {-Waist circumference } \\
\text {-Waist-hip ratio } \\
\text {-Waist-height ratio } \\
\text {-BMl age } 20 \text { (see } \\
\text { anthropometric data) }\end{array}$ & $\begin{array}{l}\text { Low-grade inflammation: excluding measures of hsCRP } \geq 10 \mathrm{mg} / \mathrm{L} \text { in } \\
\text { order to exclude values related to potential ongoing infection. } \\
\text { Physical activity: "number of days with minimum } 30 \text { minutes of } \\
\text { physical activity per week". } \\
\text { Categories } \\
\text { Low-grade inflammation: }<1.0,1.0-2.9, \geq 3.0 \mathrm{mg} / \mathrm{L} \\
\text { Physical activity: } 0,1-2, \geq 3 \text { days/week } \\
\text { Alcohol: }</ \geq 21 / 14 \text { units/week for male/female, which was the } \\
\text { recommended safe dose in } 2010 \text {, where the DD2 began enrollment } \\
\text { Waist circumference: see below } \\
\text { Waist-hip ratio: see below } \\
\text { Waist-height ratio: see below }\end{array}$ \\
\hline $\begin{array}{l}\text { Questionnaire } 2016 \text { variables } \\
\text {-Smoking } \\
\text {-Physical activity } \\
\text {-BMI }\end{array}$ & $\begin{array}{l}\text { Physical activity: "number of days with minimum } 30 \text { minutes of } \\
\text { physical activity per week". } \\
\text { Categories: } \\
\text { Smoking: Never, former, current (daily + occasionally) } \\
\text { Physical activity: } 0,1-2, \geq 3 \text { days/week } \\
\text { BMl: see below }\end{array}$ \\
\hline \multicolumn{2}{|l|}{ Anthropometric data } \\
\hline Height & $\begin{array}{l}\text { Data on height is available from } 3 \text { different sources: } \\
\text { DD2 enrollment ( } 2016 \text { onwards), DDDA data (repeated measures), } \\
\text { questionnaire data } 2016 \text { (self-reported). } \\
\text { Regarding DDDA data: a mean height based on all available DDDA } \\
\text { heights where calculated for all patients } 18 \text { years or older. } \\
\text { Heights below } 130 \mathrm{~cm} \text { and above } 220 \text { were considered outliers and } \\
\text { not included in any calculations. } \\
\text { See variables below for hierarchically order of the height }\end{array}$ \\
\hline
\end{tabular}




\begin{tabular}{|c|c|}
\hline BMI age 20 years & $\begin{array}{l}\text { Weight: } \\
\text { Recalled at DD2 enrollment. Weights below } 35 \text { and above } 300 \mathrm{~kg} \\
\text { were considered outliers and not included in the BMI calculation. } \\
\text { Height: } \\
\text { We do not expect height to change over time among these adults. } \\
\text { Thus, we used the available heights in a hierarchically order; height } \\
\text { from questionnaire survey in } 2016 \text {, DD2 enrollment, DDDA. That is, } \\
\text { if a patient has a height recorded from the questionnaire, we will } \\
\text { use that height. If not, we will use the DD2 enrollment height } \\
\text { (measured by health personal, but only a few available measures } \\
\text { [not part of the DD2 core data initially]) and if no DD2 enrollment } \\
\text { height is available, we will use DDDA height. } \\
\text { Categories: } \\
\text { BMl age } 20 \text { years: }<25,25-29,30-34, \geq 35 \mathrm{~kg} / \mathrm{m}^{2}\end{array}$ \\
\hline BMI baseline & $\begin{array}{l}\text { Weight: } \\
\text { If weight recorded as part of the DD2 enrollment process is } \\
\text { available (few [not part of the DD2 core data initially]), we used that } \\
\text { weight measure, otherwise the DDDA weight. } \\
\text { Weights below } 35 \text { and above } 300 \mathrm{~kg} \text { were considered outliers and } \\
\text { not included in the BMI calculation. } \\
\text { Height: } \\
\text { We do not expect height to change over time among these adults. } \\
\text { Thus, we used the available heights in a hierarchically order; height } \\
\text { from questionnaire survey in } 2016, \text { DD2 enrollment, DDDA. That is, } \\
\text { if a patient has a height recorded from the questionnaire, we will } \\
\text { use that height. If not, we will use the DD2 enrollment height } \\
\text { (measured by health personal, but only a few available measures } \\
\text { [not part of the DD2 core data initially]) and if no DD2 enrollment } \\
\text { height is available, we will use DDDA height. } \\
\text { Categories: } \\
\text { BMl at DD2 enrollment: }<25,25-29,30-34, \geq 35 \mathrm{~kg} / \mathrm{m}^{2}\end{array}$ \\
\hline BMI questionnaire 2016 & $\begin{array}{l}\text { Will be based solely on the weight and height data from the } 2016 \\
\text { questionnaire survey in order to report the exact same number of } \\
\text { missing as in other paper based solely on the neuropathy } \\
\text { questionnaire data. (That means } 90 \text { missing vs. } 73 \text { with if we } \\
\text { instead had used the height variable that was used in calculation of } \\
\text { the other anthropometric variables) } \\
\text { Categories: } \\
\text { BMl at DD2 enrollment: }<25,25-29,30-34, \geq 35 \mathrm{~kg} / \mathrm{m}^{2}\end{array}$ \\
\hline Waist circumference & $\begin{array}{l}\text { Categories: } \\
\text { Male/female: }<94 / 80 \mathrm{~cm}, 94-101 / 80-87 \mathrm{~cm}, \geq 102 / 88 \mathrm{~cm} \\
\text { Reference: } \\
\text { WHO: World health Organ Tech Rep Ser 2000;894:i-xii, 1-253 } \\
\text { IDF: Diabet Med 2006;23:469-480 }\end{array}$ \\
\hline
\end{tabular}




\begin{tabular}{|c|c|}
\hline Waist-hip ratio & $\begin{array}{l}\text { Categories: } \\
\text { Male/female: } \\
<0.95 / 0.85 \\
0.95-0.99 / 0.85-0.89 \\
1.00-1.04 / 0.90-0.94 \\
\geq 1.05 / 0.95 \\
\text { References: } \\
\text { We based our categories on often used waist-hip ratio } \\
\text { classifications in the scientific literature together with observations } \\
\text { of baseline WHR distributions in our cohort. }\end{array}$ \\
\hline Waist-height ratio & $\begin{array}{l}\text { Categories: } \\
<0.5,0.5-0.6, \geq 0.6 \\
\text { Reference: } \\
\text { Schneider et al. J Clin Endocrinol Metab 2007;92(2):589-594 }\end{array}$ \\
\hline Prescription data & $\begin{array}{l}\text { For all prescription data the relevant time period is around baseline } \\
=\mathrm{DD} 2 \text { enrollment. } \\
\text { Thus, lookback period is } 1 \text { year prior to DD2 enrollment date }\end{array}$ \\
\hline Lipidlowering drugs & ATC: C10 \\
\hline Antihypertensives & ATC: C02, C03, C07, C08, C09 \\
\hline Glucose-lowering drugs & $\begin{array}{l}\text { ATC: A10 } \\
\text { Categories: } \\
\text { No GLD: no A10 prescription redemption } \\
\text { Non-insulin only: } \geq 1 \text { prescription redemption of } A 10 B \text { and NO } \\
\text { prescription redemption of } A 10 A \\
\text { Insulin only: } \geq 1 \text { prescription redemption of } A 10 A \text { and NO } \\
\text { prescription redemption of } A 10 B \\
\text { Non-insulin + insulin: } \geq 1 \text { prescription redemption of } A 10 B \text { and } \geq 1 \\
\text { prescription redemption of } A 10 A\end{array}$ \\
\hline Diabetes duration & $\begin{array}{l}\text { Determined as the period time following either i) a first-time } \\
\text { prescription redemption of a glucose-lowering drug, ii) a hospital } \\
\text { record of diabetes, iii) an } \mathrm{HbA} 1 \mathrm{c} \geq 48 \mathrm{mmol} / \mathrm{mol} \text { (DDDA data), or iv) } \\
\text { time of DD2-enrolment. }\end{array}$ \\
\hline Metabolic syndrome & $\begin{array}{l}\text { Defined using the International Diabetes Federation metabolic } \\
\text { syndrome definition: } \\
\text { Metabolic syndrome: } \\
\text { Central obesity (defined as waist circumference } \geq 94 / 80 \mathrm{~cm} \\
\text { [male/female], i.e. European ethnic specific value]) AND any two of } \\
\text { the following: } \\
\text { a) Raised triglycerides } \geq 1.7 \mathrm{mmol} / \mathrm{L} \text {, or treatment for this lipid } \\
\text { abnormality. } \\
\text { b) Reduced } \mathrm{HDL} \text { cholesterol }<1.03 \mathrm{mmol} / \mathrm{L} \text { (males) } / 1.29 \mathrm{mmol} / \mathrm{mol} \\
\text { (females), or treatment for this lipid abnormality. } \\
\text { c) Raised blood pressure: systolic } \geq 130 \mathrm{mmHg} \text { or diastolic } \geq 85 \\
\text { mmHg, or treatment for hypertension. } \\
\text { d) Raised fasting plasma glucose } \geq 5.6 \mathrm{mmol} / \mathrm{L} \text { or diagnosed type } 2 \\
\text { diabetes. } \\
\text { Reference: }\end{array}$ \\
\hline
\end{tabular}


International Diabetes federation, https://www.idf.org/e-

library/consensus-statements/60-idfconsensus-worldwide-

definitionof-the-metabolic-syndrome.html 


\begin{tabular}{|c|c|c|c|c|c|}
\hline & \multirow[t]{2}{*}{ All, $\mathrm{N}=5,249$} & \multirow[t]{2}{*}{ MNSIq $<4, N=4,311$} & \multirow[t]{2}{*}{$\mathrm{MNSI} \geq 4, \mathrm{~N}=938$} & \multicolumn{2}{|c|}{ MNSIq $\geq 4$} \\
\hline & & & & $\begin{array}{l}\text { No pain or DN4 <3, } \\
N=552\end{array}$ & $\begin{array}{l}\text { Pain and DN4 } \geq 3 \text {, } \\
N=386\end{array}$ \\
\hline & Total & No DPN & DPN & Non-painful DPN & Painful DPN \\
\hline \multicolumn{6}{|l|}{ Total cohort, $\mathrm{N}=5249$} \\
\hline Total, $\mathrm{N}$ & 5249 & $4311(82.1)$ & $938(17.9)$ & $552(10.5)$ & $386(7.4)$ \\
\hline Age, questionnaire 2016, N=5249 & $65.4(56.6 ; 71.5)$ & $65.8(57.2 ; 71.7)$ & $63.1(54.9 ; 70.3)$ & $62.8(54.9 ; 70.0)$ & $64.0(55.3 ; 70.8)$ \\
\hline Female sex, $\mathrm{N}=5249$ & $2216(42.2)$ & $1770(41.1)$ & $446(47.5)$ & $258(46.7)$ & $188(48.7)$ \\
\hline $\begin{array}{l}\text { Diabetes duration, questionnaire 2016, years, } \\
N=5247\end{array}$ & $4.6(3.5 ; 5.7)$ & $4.5(3.4 ; 5.7)$ & $4.8(3.7 ; 6.0)$ & $4.7(3.6 ; 5.9)$ & $4.9(3.8 ; 6.1)$ \\
\hline Height, $\mathrm{N}=5220$ & $173(166 ; 180)$ & $173(166 ; 179)$ & $172(165 ; 180)$ & $172(165 ; 180)$ & $173(166 ; 180)$ \\
\hline BMI, age 20 years, $\mathrm{kg} / \mathrm{m} 2, \mathrm{~N}=4542$ & $23.2(21.0 ; 25.5)$ & $23.2(21.0 ; 25.5)$ & $23.2(20.9 ; 25.7)$ & $23.3(21.1 ; 25.8)$ & $23.1(20.5 ; 25.2)$ \\
\hline BMI, questionnaire 2016, kg/m2, N=5159 & $29.7(26.4 ; 33.6)$ & $29.4(26.2 ; 33.1)$ & $31.2(27.7 ; 35.7)$ & $31.2(27.8 ; 35.5)$ & $31.5(27.5 ; 35.7)$ \\
\hline Waist circumference, $\mathrm{cm}$, baseline, $\mathrm{N}=5239$ & $106(97 ; 116)$ & $105(96 ; 115)$ & $110(100 ; 119)$ & $110(100 ; 120)$ & $110(100 ; 119)$ \\
\hline Waist-hip ratio, baseline, $\mathrm{N}=5237$ & $0.98(0.92 ; 10.4)$ & $0.98(0.92 ; 1.04)$ & $0.98(0.92 ; 1.04)$ & $0.98(0.92 ; 1.04)$ & $0.99(0.93 ; 1.04)$ \\
\hline Waist-height ratio, $\mathrm{N}=5210$ & $0.61(0.56 ; 0.67)$ & $0.61(0.56 ; 0.66)$ & $0.63(0.58 ; 0.69)$ & $0.63(0.58 ; 0.70)$ & $0.64(0.58 ; 0.69)$ \\
\hline Low-grade inflammation (hsCRP), ${ }^{*} \mathrm{mg} / \mathrm{L}, \mathrm{N}=4722$ & $1.7(0.8 ; 3.4)$ & $1.6(0.7 ; 3.1)$ & $2.2(1.0 ; 4.2)$ & $2.2(0.9 ; 4.2)$ & $2.2(1.0 ; 4.2)$ \\
\hline C-peptide, $\mathrm{pmol} / \mathrm{L}, \mathrm{N}=4353$ & $1149(856 ; 1553)$ & $1128(841 ; 1516)$ & $1266(958 ; 1701)$ & $1256(927 ; 1691)$ & $1276(978 ; 1717)$ \\
\hline Physical activity, $\uparrow$ baseline, days/week, N=5247 & $4(2 ; 7)$ & $4(2 ; 7)$ & $3(1 ; 7)$ & $3(1 ; 7)$ & $3(1 ; 7)$ \\
\hline $\begin{array}{l}\text { Physical activity, } \uparrow \text { questionnaire } 2116 \text {, days/week, } \\
\end{array}$ & $4(2 ; 6)$ & $4(2 ; 6)$ & $3(1 ; 6)$ & $3(2 ; 5)$ & $3(1 ; 6)$ \\
\hline Alcohol, baseline, $\mathrm{N}=5247$ & & & & & \\
\hline > 21/14 units/week (male/female) & $343(6.5)$ & $277(6.4)$ & $66(7.0)$ & $32(5.8)$ & $34(8.8)$ \\
\hline \multicolumn{6}{|l|}{ Smoking, questionnaire $2016, \mathrm{~N}=5231$} \\
\hline Never & $1850(35.4)$ & $1584(36.9)$ & $266(28.4)$ & $174(31.5)$ & $92(23.8)$ \\
\hline Former & $2361(45.1)$ & 1909 (44.5) & $452(48.2)$ & $253(45.8)$ & $199(51.6)$ \\
\hline Current & $1020(19.5)$ & $800(18.6)$ & $220(23.5)$ & $125(22.6)$ & $95(24.6)$ \\
\hline Antihypertensive drug use, $\mathrm{N}=5247$ & $3874(73.8)$ & $3147(73,0)$ & $727(77.5)$ & $426(77.2)$ & $301(78.0)$ \\
\hline Lipid lowering drug use, $\mathrm{N}=5247$ & $3764(71,7)$ & $3102(72.0)$ & $662(70.6)$ & $396(71.7)$ & $266(68.9)$ \\
\hline \multicolumn{6}{|l|}{ Glucose-lowering drug use, $\mathrm{N}=5247$} \\
\hline Any glucose-lowering drug & $4460(85.0)$ & $3640(84.5)$ & $820(87.4)$ & $477(86.4)$ & $343(88.9)$ \\
\hline Non-insulin glucose-lowering drug only & $4143(79.0)$ & $3408(79.1)$ & $735(78.4)$ & $432(78.3)$ & $303(78.5)$ \\
\hline Insulin only & $53(1.0)$ & $39(0.9)$ & $14(1.5)$ & $7(1.3)$ & $7(1.8)$ \\
\hline Both insulin + non-insulin glucose-lowering drug & $264(5.0)$ & $193(4.5)$ & $71(7.6)$ & $38(6.9)$ & $33(8.6)$ \\
\hline \multicolumn{6}{|l|}{ Subcohort, $\mathrm{N}=3,623$} \\
\hline BMI, baseline, $\mathrm{kg} / \mathrm{m} 2, \mathrm{~N}=3263$ & $30.3(27.1 ; 34.3)$ & $30.0(26.8 ; 34.0)$ & $31.8(28.1: 36.0)$ & $32.0(28.4: 36.0)$ & $31.5(27.7: 36.0)$ \\
\hline Systolic blood pressure, $\mathrm{mmHg}, \mathrm{N}=3387$ & $130(124 ; 140)$ & $130(124 ; 140)$ & $130(124: 140)$ & 130 (123: 140) & 130 (124: 142) \\
\hline Diastolic blood pressure, $\mathrm{mmHg}, \mathrm{N}=3387$ & $80(75 ; 86)$ & $80(74 ; 85)$ & $80(75 ; 86)$ & $80(75 ; 87)$ & $80(75 ; 86)$ \\
\hline \multicolumn{6}{|l|}{ Dyslipidemia } \\
\hline Total cholesterol, $\mathrm{mmol} / \mathrm{L}, \mathrm{N}=2267$ & $4.3(3.7 ; 5.1)$ & $4.3(3.7 ; 5.1)$ & $4.4(3.8 ; 5.1)$ & $4.3(3.7 ; 5.0)$ & $4.5(3.9 ; 5.3)$ \\
\hline HDL cholesterol, $\mathrm{mmol} / \mathrm{L}, \mathrm{N}=2274$ & $1.2(1.0 ; 1.5)$ & $1.2(1.0 ; 1.5)$ & $1.2(1.0 ; 1.4)$ & $1.2(1.0 ; 1.4)$ & $1.2(1.0 ; 1.4)$ \\
\hline $\mathrm{LDL}, \mathrm{mmol} / \mathrm{L}, \mathrm{N}=3433$ & $2.2(1.7 ; 2.8)$ & $2.2(1.7 ; 2.8)$ & $2.2(1.7 ; 2.9)$ & $2.2(1.7 ; 2.8)$ & $2.3(1.8 ; 2.9)$ \\
\hline Triglycerides, $\mathrm{mmol} / \mathrm{L}, \mathrm{N}=3302$ & $1.6(1.1 ; 2.3)$ & $1.6(1.1 ; 2.3)$ & $1.9(1.3 ; 2.6)$ & $1.8(1.2 ; 2.5)$ & $1.9(1.3 ; 2.9)$ \\
\hline Glycemic control (HbA1c), mmol/mol, $\mathrm{N}=3536$ & $47(43 ; 53)$ & $47(43 ; 53)$ & $48(42 ; 56)$ & $47(42 ; 57)$ & $48(43 ; 55)$ \\
\hline Glycemic control (HbA1c), \%, N=3536 & $6.5(6.1 ; 7.0)$ & $6.5(6.1 ; 7.0)$ & $6.5(6.0 ; 7.3)$ & $6.5(6.0 ; 7.4)$ & $6.5(6.1 ; 7.2)$ \\
\hline \multicolumn{6}{|l|}{ Smoking, baseline, $\mathrm{N}=3437$} \\
\hline Never & $1643(47.8)$ & $1398(49.4)$ & $245(40.3)$ & $154(42.9)$ & $91(36.6)$ \\
\hline Former & $1189(34.6)$ & $960(33.9)$ & $229(37.7)$ & $128(35.7)$ & $101(40.6)$ \\
\hline Current & $605(17.6)$ & $471(16.7)$ & $134(22.0)$ & $77(21.5)$ & $57(22.9)$ \\
\hline
\end{tabular}


Abbreviations: MNSlq; Michigan Neuropathy Screening Instrument questionnaire, DN4; Douleur Neuropathique en 4 questions, DPN; diabetic polyneuropathy, DD2; The Danish Centre for Strategic Research in Type 2 Diabetes, DDDA; Danish Diabetes Database for Adults, BMl; body mass index, hsCRP; highsensitivity C-reactive protein, $\mathrm{HbA1c}$; hemoglobin A1c.

${ }^{*}$ Of note, CRP value was available for 5111 , of which 389 had hsCRP $\geq 10 \mathrm{mg} / \mathrm{L}$

†Days per week with minimum 30 minutes of physical activity

Missing data in the total cohort $(n=5249)$; height $(n=29$ missing); BMl at 20 years $(n=707$ missing); BMl at questionnaire 2016 ( $n=90)$; waist circumference ( $n=10$ missing); waist-hip ratio ( $n=12$ missing), waist-height ratio ( $n=39$ missing), low-grade inflammation ( $n=138$ missing hsCRP, 389 with hsCRP $\geq 10$ $\mathrm{mg} / \mathrm{L})$; C-peptide ( $\mathrm{n}=896$ missing); physical activity at baseline $(\mathrm{n}=2$ missing); physical activity at questionnaire $2016(\mathrm{n}=60)$, alcohol at baseline $(\mathrm{n}=2$ missing), alcohol at questionnaire $2016(n=70)$, smoking at questionnaire $(n=018)$, remaining variables $(n=0$ missing)

Missing data in the DDDA-subcohort $(n=3623)$ : BMl at baseline $(n=360)$; blood pressure $(n=236$ missing); total cholesterol $(n=1356$ missing); HDL cholesterol ( $n=1,349$ missing); LDL cholesterol ( $n=190$ missing); triglycerides $(n=321$ missing); HbA1c $(n=87$ missing); albumin/creatinine ratio ( $n=0$ missing), smoking at baseline ( $\mathrm{n}=186$ missing). 


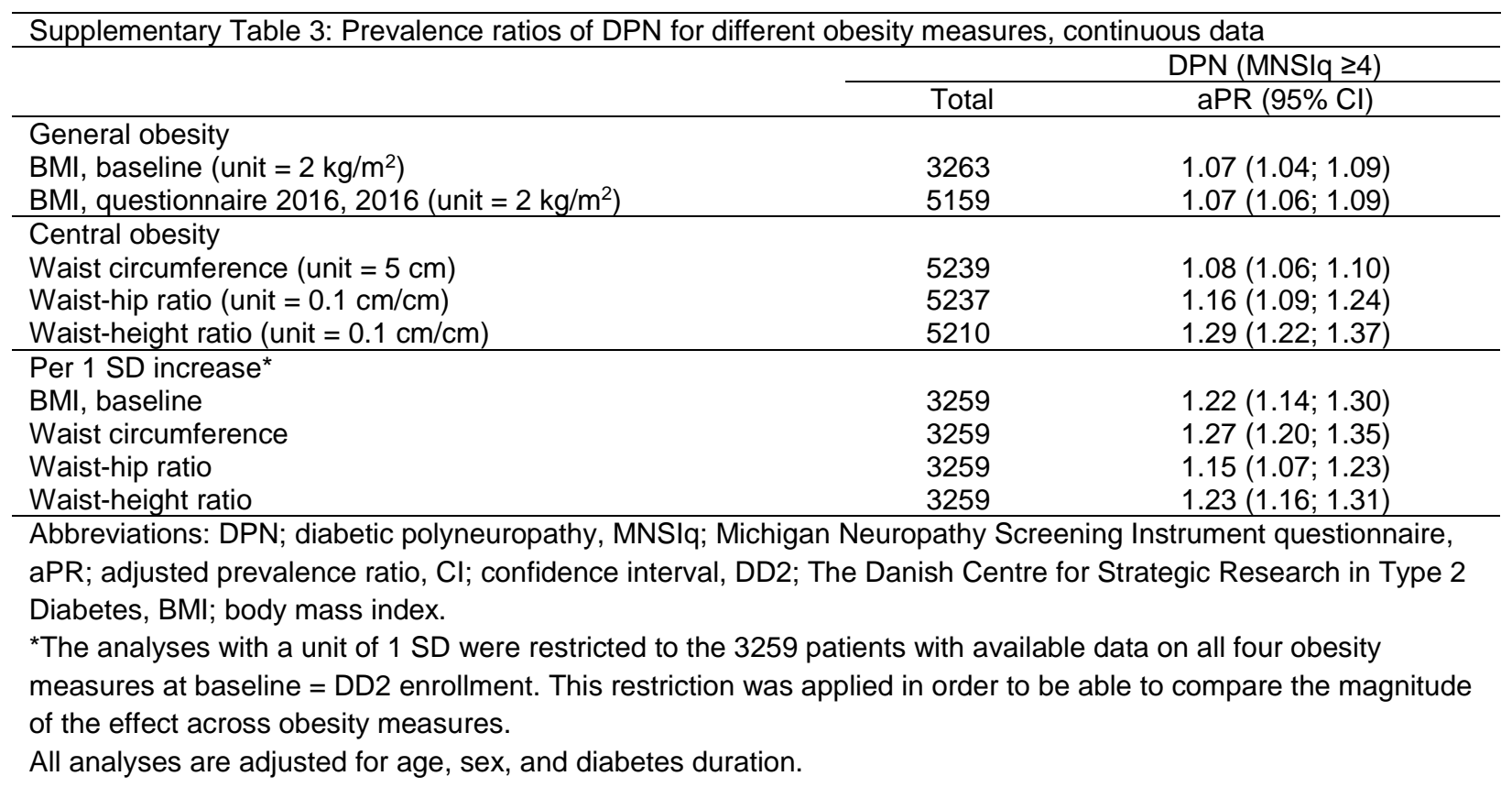




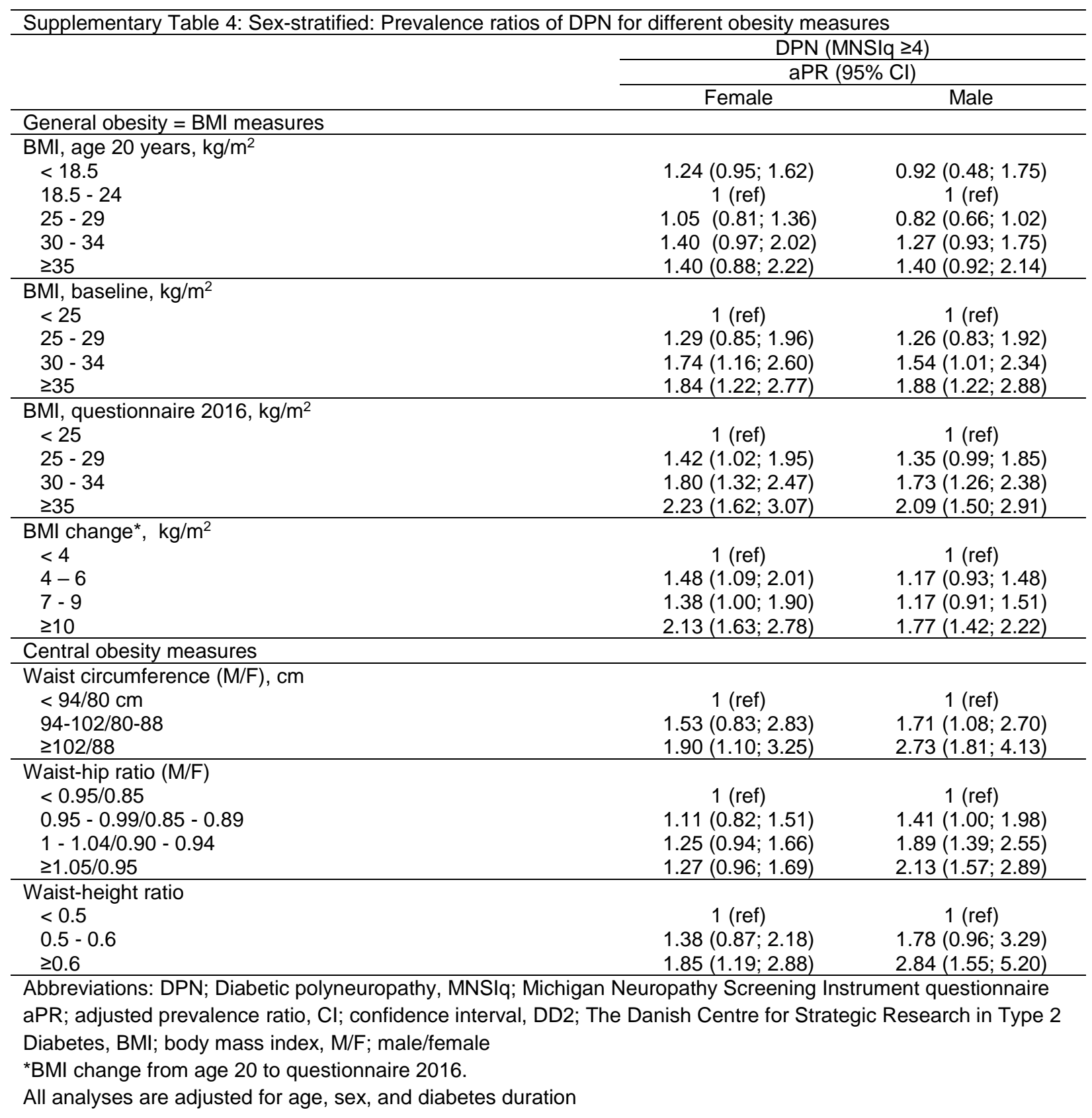




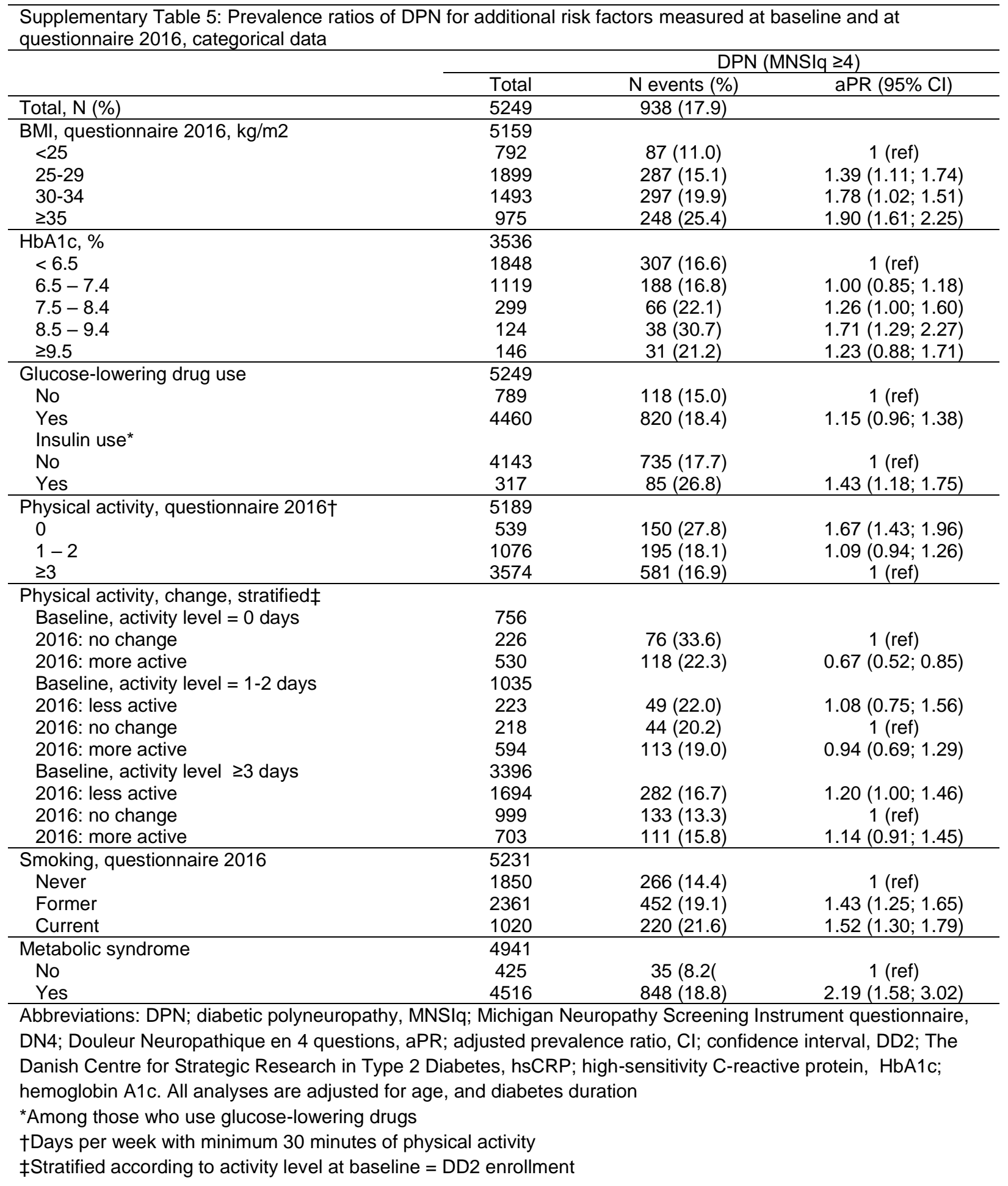




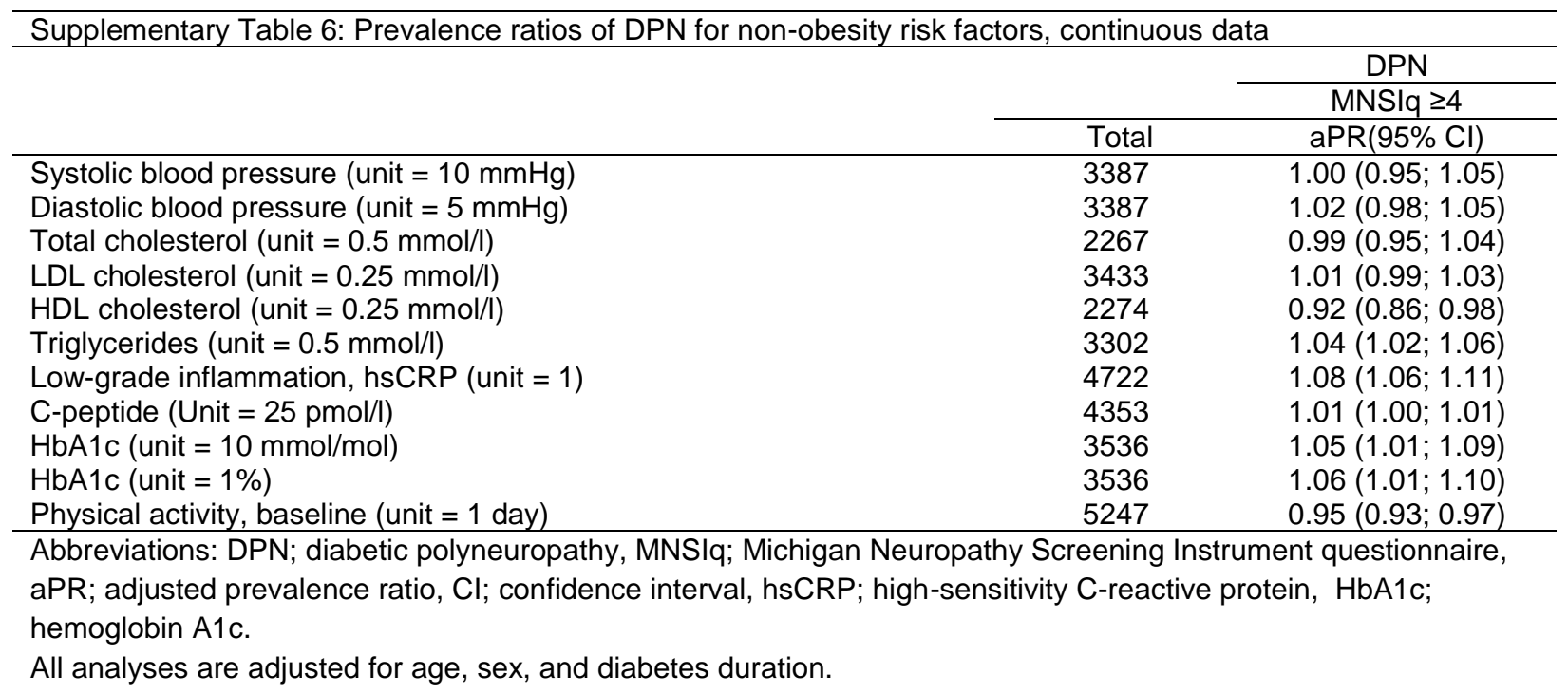




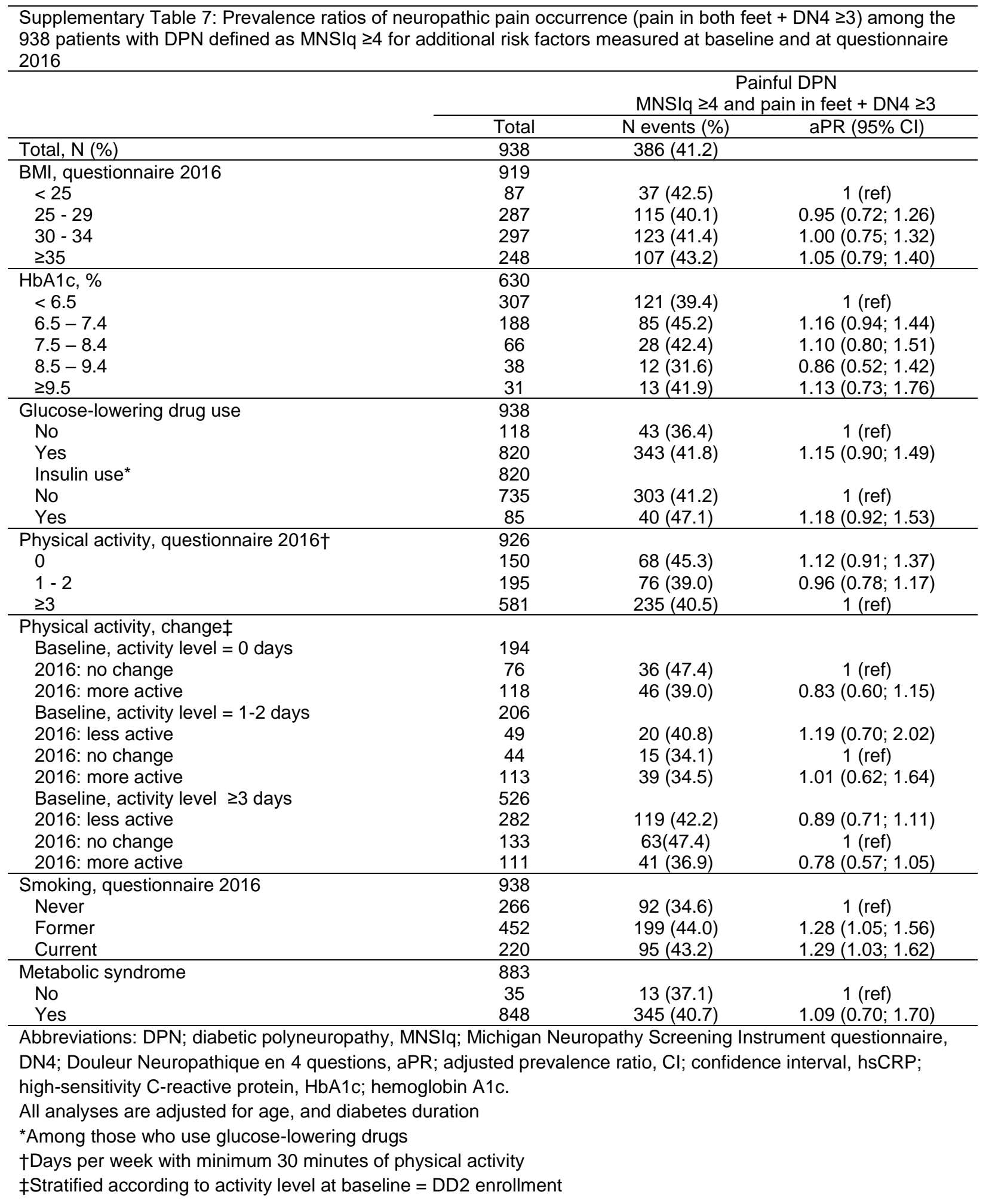




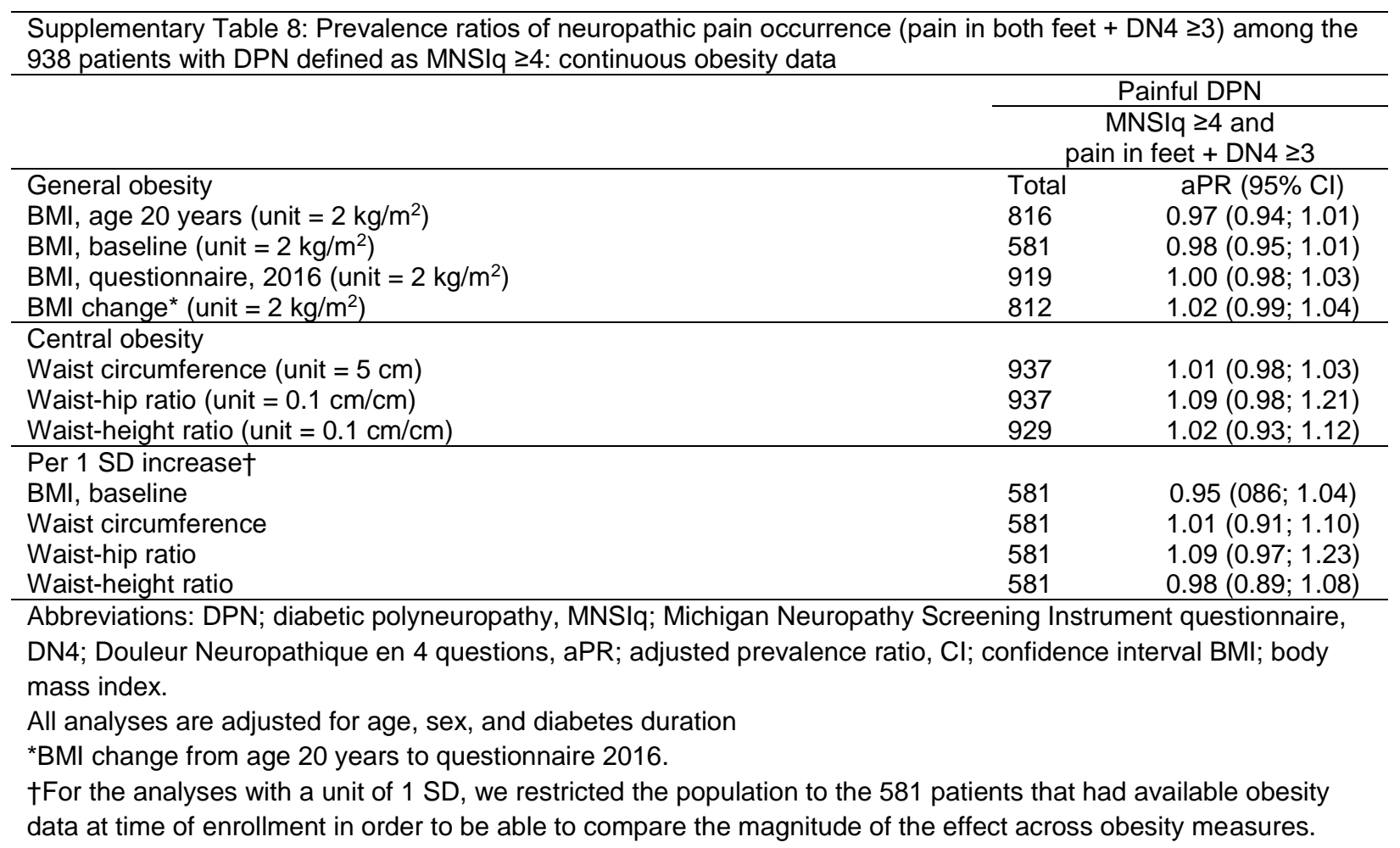




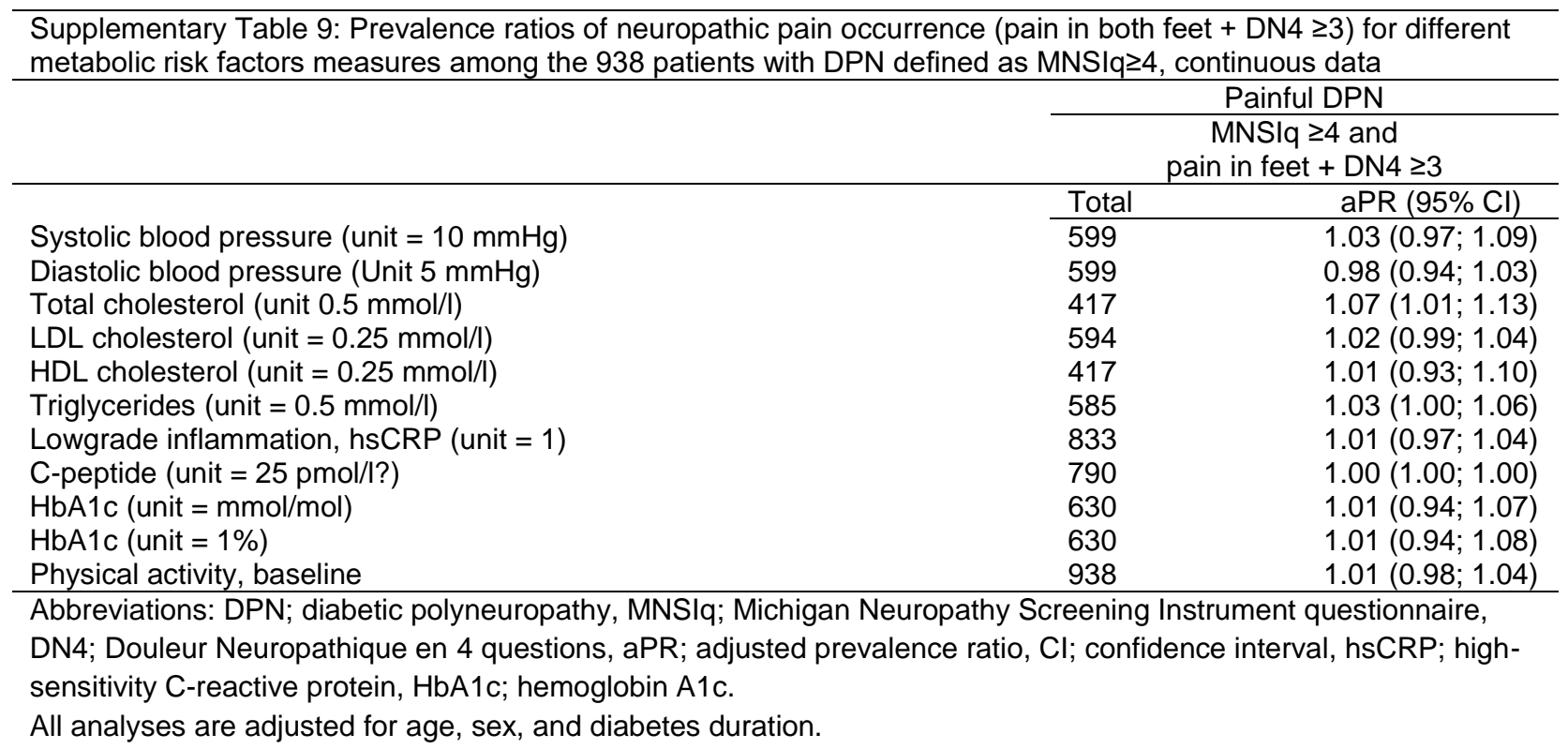




\begin{tabular}{|c|c|c|}
\hline \multicolumn{3}{|c|}{$\begin{array}{l}\text { Supplementary Table } 10 \text { : Sex-stratified analyses: Prevalence ratios of neuropathic pain occurrence (pain in botl } \\
\text { feet + DN4 } \geq 3 \text { ) among the } 938 \text { patients with DPN defined as MNSIa } \geq 4 \text { for obesity measures. }\end{array}$} \\
\hline & Female & Male \\
\hline & $\begin{array}{c}\text { aPR } \\
(95 \% \mathrm{Cl})\end{array}$ & $\begin{array}{c}\text { aPR } \\
(95 \% \mathrm{Cl})\end{array}$ \\
\hline \multicolumn{3}{|c|}{ General obesity = BMI measures } \\
\hline \multicolumn{3}{|c|}{$\mathrm{BMl}$, age 20 years, $\mathrm{kg} / \mathrm{m}^{2}$} \\
\hline$<18.5$ & $1.13(0.81 ; 1.57)$ & $0.84(0.34 ; 2.09)$ \\
\hline $18.5-24$ & 1 (ref) & 1 (ref) \\
\hline $25-29$ & $1.01(0.71 ; 1.43)$ & $0.78(0.58 ; 1.06)$ \\
\hline $30-34$ & $0.93(0.55 ; 1.58)$ & $0.80(0.49 ; 1.29)$ \\
\hline$\geq 35$ & $0.64(0.27 ; 1.53)$ & $0.90(0.51 ; 1.68)$ \\
\hline \multicolumn{3}{|l|}{ BMI, baseline, $\mathrm{kg} / \mathrm{m}^{2}$} \\
\hline$<25$ & 1 (ref) & 1 (ref) \\
\hline $25-29$ & $0.69(0.44 ; 1.07)$ & $0.82(0.50 ; 1.34)$ \\
\hline $30-34$ & $0.68(0.45 ; 1.04)$ & $0.88(0.54 ; 1.44)$ \\
\hline$\geq 35$ & $0.66(0.43 ; 1.02)$ & $0.86(0.51 ; 1.43)$ \\
\hline \multicolumn{3}{|c|}{ BMI, questionnaire, 2016, $\mathrm{kg} / \mathrm{m}^{2}$} \\
\hline$<25$ & 1 (ref) & 1 (ref) \\
\hline $25-29$ & $0.91(0.61 ; 1.34)$ & $1.00(0.66 ; 1.52)$ \\
\hline $30-34$ & $0.94(0.64 ; 1.37)$ & $1.06(0.70 ; 1.61)$ \\
\hline$\geq 35$ & $1.00(0.68 ; 1.34)$ & $1.12(0.72 ; 1.73)$ \\
\hline \multicolumn{3}{|l|}{ BMI change $^{*}, \mathrm{~kg} / \mathrm{m}^{2}$} \\
\hline$<4$ & 1 (ref) & 1 (ref) \\
\hline $4-6$ & $1.05(0.68 ; 1.62)$ & $1.15(0.85 ; 1.56)$ \\
\hline $7-9$ & $1.33(0.88 ; 2.01)$ & $1.12(0.80 ; 1.58)$ \\
\hline$\geq 10$ & $1.14(0.78 ; 1.67)$ & $1.16(0.86 ; 1.58)$ \\
\hline \multicolumn{3}{|c|}{ Central obesity } \\
\hline \multicolumn{3}{|c|}{ Waist circumference $(M / F), \mathrm{cm}$} \\
\hline$<94 / 80 \mathrm{~cm}$ & 1 (ref) & 1 (ref) \\
\hline $94-102 / 80-88 \mathrm{~cm}$ & $1.24(0.51 ; 3.02)$ & $1.56(0.75 ; 3.24)$ \\
\hline$\geq 102 / 88 \mathrm{~cm}$ & $1.27(0.57 ; 2.85)$ & $1.50(0.75 ; 3.00)$ \\
\hline \multicolumn{3}{|l|}{ Waist-hip ratio $(\mathrm{M} / \mathrm{F})$} \\
\hline$<0.95 / 0.85$ & 1 (ref) & 1 (ref) \\
\hline $0.95-0.99 / 0.85-0.89$ & $0.82(0.54 ; 1.26)$ & $1.85(1.09 ; 3.15)$ \\
\hline $1-1.04 / 0.90-0.94$ & $1.00(0.69 ; 1.46)$ & $1.50(0.89 ; 2.52)$ \\
\hline$\geq 1.05 / 0.95$ & $1.16(0.81 ; 1.66)$ & $1.61(0.96 ; 2.70)$ \\
\hline \multicolumn{3}{|l|}{ Waist-height ratio } \\
\hline$<0.5$ & 1 (ref) & 1 (ref) \\
\hline $0.5-0.6$ & $0.76(0.46 ; 1.27)$ & $1.36(0.52 ; 3.59)$ \\
\hline$\geq 0.6$ & $0.87(0.54 ; 1.41)$ & $1.37(0.53 ; 3.57)$ \\
\hline
\end{tabular}

Abbreviations: DPN; Diabetic polyneuropathy, MNSlq; Michigan Neuropathy Screening Instrument questionnaire aPR; adjusted prevalence ratio, $\mathrm{Cl}$; confidence interval, $\mathrm{BMI}$; body mass index, M/F; male/female All analyses are adjusted for age, sex, and diabetes duration *BMl change from age 20 years to questionnaire 2016. 


\begin{tabular}{|c|c|c|c|c|}
\hline & $\begin{array}{l}\text { Main results } \\
\text { adjusted for } \\
\text { age, sex, and } \\
\text { diabetes } \\
\text { duration }\end{array}$ & $\begin{array}{l}\text { Additional } \\
\text { adjusted for } \\
\text { waist-hip ratio }\end{array}$ & $\begin{array}{l}\text { Additional } \\
\text { adjusted for } \\
\text { HbA1c }\end{array}$ & $\begin{array}{c}\text { Additional } \\
\text { adjusted for } \\
\text { waist-hip ratio } \\
\text { and } \mathrm{HbA1c}\end{array}$ \\
\hline & aPR $(95 \% \mathrm{Cl})$ & aPR $(95 \% \mathrm{Cl})$ & aPR $(95 \% \mathrm{Cl})$ & aPR $(95 \% \mathrm{Cl})$ \\
\hline \multicolumn{5}{|l|}{$\begin{array}{l}\text { NON-OBESITY METABOLIC } \\
\text { AND LIFESTYLE FACTORS AT } \\
\text { BASELINE }\end{array}$} \\
\hline $\begin{array}{l}\text { Blood pressure (systolic) } \mathrm{mmHg} \\
<130 \\
\geq 130\end{array}$ & $\begin{array}{c}1 \text { (ref) } \\
0.95(0.82 ; 1.10)\end{array}$ & $\begin{array}{c}1 \text { (ref) } \\
0.93(0.80 ; 1.08)\end{array}$ & $\begin{array}{c}1 \text { (ref) } \\
0.94(0.81 ; 1.09)\end{array}$ & $\begin{array}{c}1 \text { (ref) } \\
0.93(0.80 ; 1.08)\end{array}$ \\
\hline $\begin{array}{l}\text { Blood pressure (diastolic) } \mathrm{mmHg} \\
<80 \\
\geq 80\end{array}$ & $\begin{array}{c}1 \text { (ref) } \\
1.10(0.95 ; 1.29)\end{array}$ & $\begin{array}{c}1 \text { (ref) } \\
1.09(0.94 ; 1.27)\end{array}$ & $\begin{array}{c}1 \text { (ref) } \\
1.09(0.93 ; 1.27)\end{array}$ & $\begin{array}{c}1 \text { (ref) } \\
1.08(0.92 ; 1.25)\end{array}$ \\
\hline $\begin{array}{l}\text { Antihypertensive drug use } \\
\text { No } \\
\text { Yes }\end{array}$ & $\begin{array}{c}1 \text { (ref) } \\
1.34(1.16 ; 1.55)\end{array}$ & $\begin{array}{c}1 \text { (ref) } \\
1.30(1.12 ; 1.50)\end{array}$ & $\begin{array}{c}1 \text { (ref) } \\
1.34(1.12 ; 1.61)\end{array}$ & $\begin{array}{c}1 \text { (ref) } \\
1.31(1.09: 1.58)\end{array}$ \\
\hline $\begin{array}{l}\text { Total cholesterol, } \mathrm{mmol} / \mathrm{L} \\
<4.3 \\
\geq 4.3\end{array}$ & $\begin{array}{c}1 \text { (ref) } \\
1.04(0.87 ; 1.24)\end{array}$ & $\begin{array}{c}1 \text { (ref) } \\
1.04(0.88 ; 1.25)\end{array}$ & $\begin{array}{c}1 \text { (ref) } \\
1.04(0.87 ; 1.24)\end{array}$ & $\begin{array}{c}1 \text { (ref) } \\
1.05(0.88 ; 1.25)\end{array}$ \\
\hline $\begin{array}{l}\text { LDL cholesterol } \\
<1.8 \\
1.8-2.6 \\
\geq 2.6\end{array}$ & $\begin{array}{c}1 \text { (ref) } \\
0.97(0.80 ; 1.16) \\
1.09(0.91 ; 1.32)\end{array}$ & $\begin{array}{c}1 \text { (ref) } \\
0.96(0.80 ; 1.16) \\
1.10(0.91 ; 1.33)\end{array}$ & $\begin{array}{c}1 \text { (ref) } \\
0.97(0.81 ; 1.17) \\
1.10(0.91 ; 1.32)\end{array}$ & $\begin{array}{c}1 \text { (ref) } \\
0.97(0.81 ; 1.17) \\
1.10(0.91 ; 1.33)\end{array}$ \\
\hline $\begin{array}{l}\text { HDL cholesterol }(\mathrm{M} / \mathrm{F})) \\
<1.0 / 1.2 \\
\geq 1.0 / 1.2\end{array}$ & $\begin{array}{c}1.35(1.12 ; 1.62) \\
1 \text { (ref) }\end{array}$ & $\begin{array}{c}1.30(1.07 ; 1.57) \\
1 \text { (ref) }\end{array}$ & $\begin{array}{c}1.28(1.06 ; 1.55) \\
1 \text { (ref) }\end{array}$ & $\begin{array}{c}1.24(1.03 ; 1.50) \\
1 \text { (ref) }\end{array}$ \\
\hline $\begin{array}{l}\text { Triglycerides } \\
<1.7 \\
\geq 1.7\end{array}$ & $\begin{array}{c}1 \text { (ref) } \\
1.36(1.17 ; 1.59)\end{array}$ & $\begin{array}{c}1 \text { (ref) } \\
1.33(1.14 ; 1.55)\end{array}$ & $\begin{array}{c}1 \text { (ref) } \\
1.34(1.15 ; 1.56)\end{array}$ & $\begin{array}{c}1 \text { (ref) } \\
1.31(1.12 ; 1.53)\end{array}$ \\
\hline $\begin{array}{l}\text { Lipid-lowering drug use } \\
\text { No } \\
\text { Yes }\end{array}$ & $\begin{array}{c}1 \text { (ref) } \\
0.97(0.86 ; 1.11)\end{array}$ & $\begin{array}{c}1 \text { (ref) } \\
0.95(0.84 ; 1.09)\end{array}$ & $\begin{array}{c}1 \text { (ref) } \\
0.89(0.76 ; 1.04)\end{array}$ & $\begin{array}{c}1 \text { (ref) } \\
0.87(0.74 ; 1.02)\end{array}$ \\
\hline $\begin{array}{l}\text { Low-grade inflammation } \\
\text { (hsCRP) }^{*}, \mathrm{mg} / \mathrm{L} \\
<1.0 \\
1.0-2.9 \\
\geq 3.0\end{array}$ & $\begin{array}{c}1 \text { (ref) } \\
1.12(0.95 ; 1.31) \\
1.66(1.42 ; 1.94)\end{array}$ & $\begin{array}{c}1 \text { (ref) } \\
1.10(0.93 ; 1.29) \\
1.61(1.37 ; 1.88) \\
\end{array}$ & $\begin{array}{c}1 \text { (ref) } \\
1.23(1.01 ; 1.50) \\
1.71(1.41 ; 2.08)\end{array}$ & $\begin{array}{c}1 \text { (ref) } \\
1.21(0.99 ; 1.48) \\
1.66(1.37 ; 2.02)\end{array}$ \\
\hline $\begin{array}{l}\text { C-peptide } \\
<850 \\
850-1550 \\
\geq 1550 \\
\end{array}$ & $\begin{array}{c}1 \text { (ref) } \\
1.29(1.08 ; 1.53) \\
1.72(1.43 ; 2.07)\end{array}$ & $\begin{array}{c}1 \text { (ref) } \\
1.25(1.04 ; 1.49) \\
1.65(1.36 ; 2.00) \\
\end{array}$ & $\begin{array}{c}1 \text { (ref) } \\
1.20(0.98 ; 1.47) \\
1.57(1.27 ; 1.95) \\
\end{array}$ & $\begin{array}{c}1 \text { (ref) } \\
1.16(0.94 ; 1.42) \\
1.49(1.19 ; 1.85) \\
\end{array}$ \\
\hline $\begin{array}{l}\mathrm{HbA} 1 \mathrm{c}, \mathrm{mmol} / \mathrm{mol} \\
<48 \\
48-57 \\
58-67 \\
68-77 \\
\geq 78 \\
\end{array}$ & $\begin{array}{c}1 \text { (ref) } \\
1.00(0.85 ; 1.19) \\
1.28(1.01 ; 1.62) \\
1.61(1.20 ; 2.17) \\
1.42(1.06 ; 1.88)\end{array}$ & $\begin{array}{c}1 \text { (ref) } \\
0.99(0.84 ; 1.17) \\
1.24(0.98 ; 1.58) \\
1.58(1.17 ; 2.13) \\
1.35(1.02 ; 1.81)\end{array}$ & $\begin{array}{l}- \\
- \\
- \\
- \\
-\end{array}$ & $\begin{array}{l}- \\
- \\
- \\
- \\
-\end{array}$ \\
\hline $\begin{array}{l}\text { Physical activity, baseline } \dagger \\
0 \\
1-2 \\
\geq 3\end{array}$ & $\begin{array}{c}1.60(1.39 ; 1.85) \\
1.26(1.09 ; 1.46) \\
1 \text { (ref) }\end{array}$ & $\begin{array}{l}1.58(1.37 ; 1.82) \\
1.25(1.08 ; 1.45) \\
1 \text { (ref) }\end{array}$ & $\begin{array}{c}1.72(1.44 ; 2.04) \\
1.24(1.04 ; 1.48) \\
1 \text { (ref) }\end{array}$ & $\begin{array}{c}1.68(1.41 ; 2.00) \\
1.22(1.02 ; 1.46) \\
1 \text { (ref) } \\
\end{array}$ \\
\hline $\begin{array}{l}\text { Physical activity, change } \\
\text { Decreased activity } \\
\text { No change } \\
\text { Increased activity }\end{array}$ & $\begin{array}{c}0.97(0.83 ; 1.12) \\
1 \text { (ref) } \\
1.05(0.91 ; 1.22)\end{array}$ & $\begin{array}{c}0.97(0.83 ; 1.12) \\
1 \text { (ref) } \\
1.05(0.91 ; 1.22)\end{array}$ & $\begin{array}{c}0.95(0.79 ; 1.13) \\
1 \text { (ref) } \\
0.98(0.81 ; 1.17)\end{array}$ & $\begin{array}{c}0.94(0.79 ; 1.13) \\
1 \text { (ref) } \\
0.97(0.81 ; 1.16)\end{array}$ \\
\hline $\begin{array}{l}\text { Smoking, baseline } \\
\text { Never }\end{array}$ & 1 (ref) & 1 (ref) & 1 (ref) & 1 (ref) \\
\hline
\end{tabular}




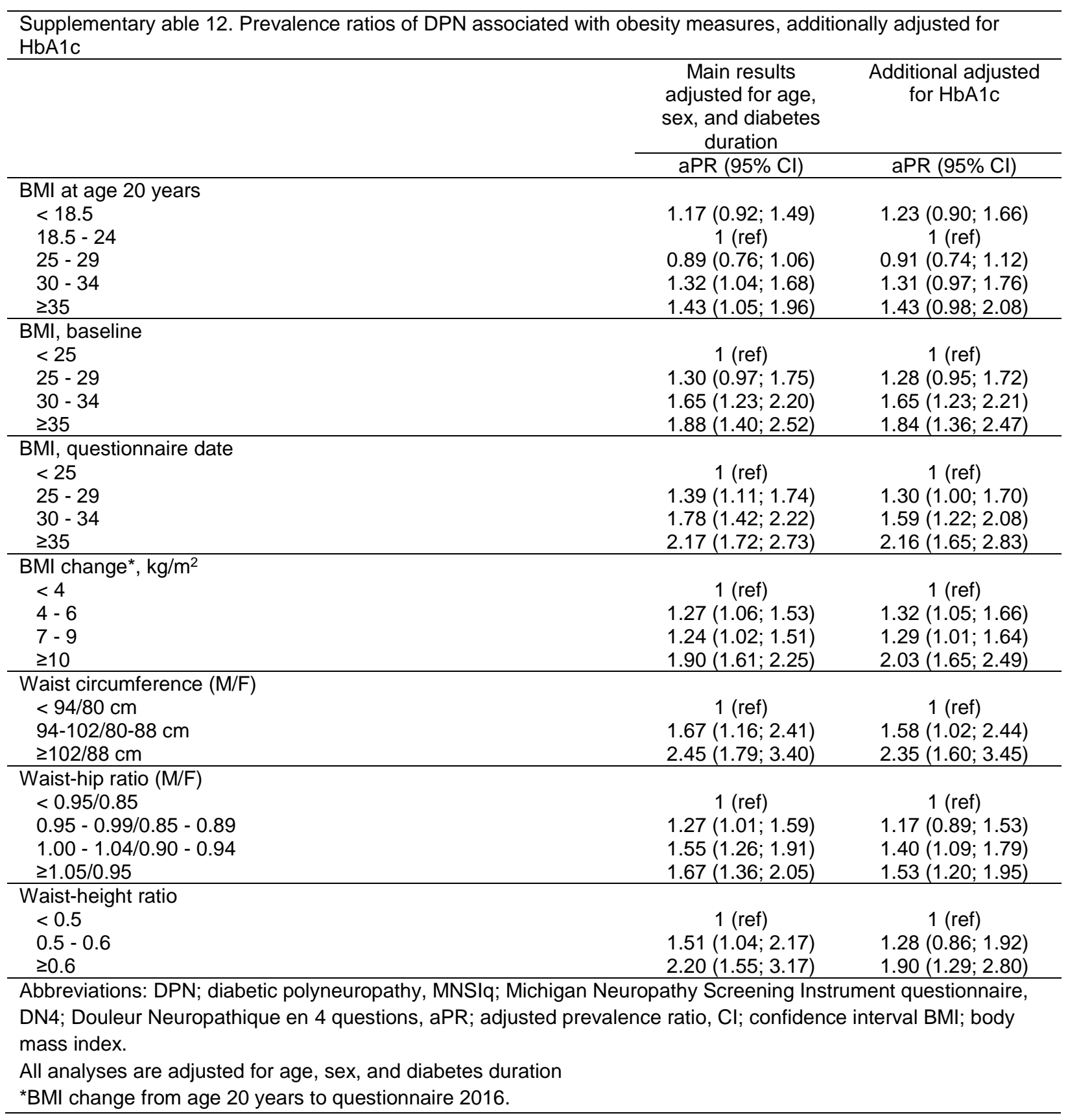




\section{Figures:}

Supplementary Figure 1: Flowchart

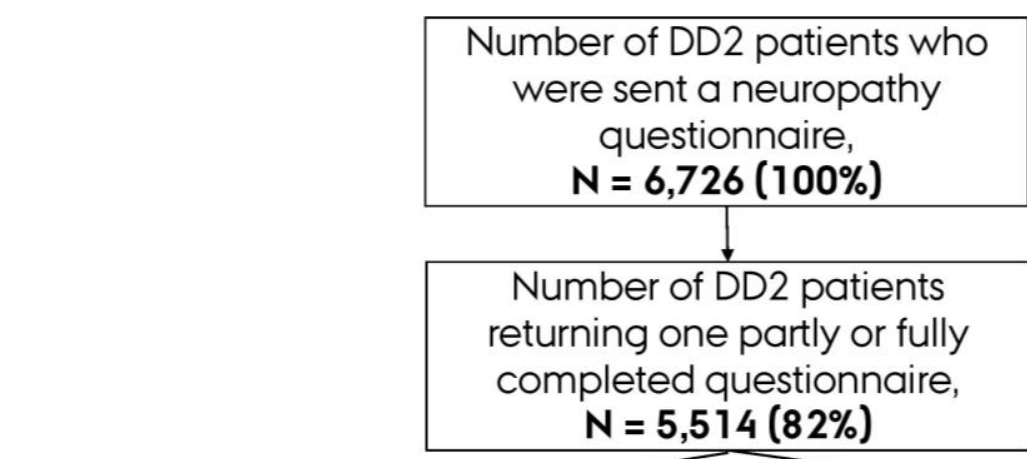

Number of DD2 patients with valid answers on the MNSIq, $\mathrm{N}=\mathbf{5 , 3 5 9}$
Number of DD2 patients with valid answers to the question on pain in both feet and the DN4 questionnaire, $\mathrm{N}=\mathbf{5 , 3 7 2}$

Number of DD2 patients with valid data on MNSlq, the question on pain in both feet, and the DN4, $N=5,249(78 \%)$ = study population 
Supplementary Figure 2: Schematic overview of the definition of DPN and the division into nonpainful DPN and painful DPN in A) main analyses and B) sensitivity analyses.

A)

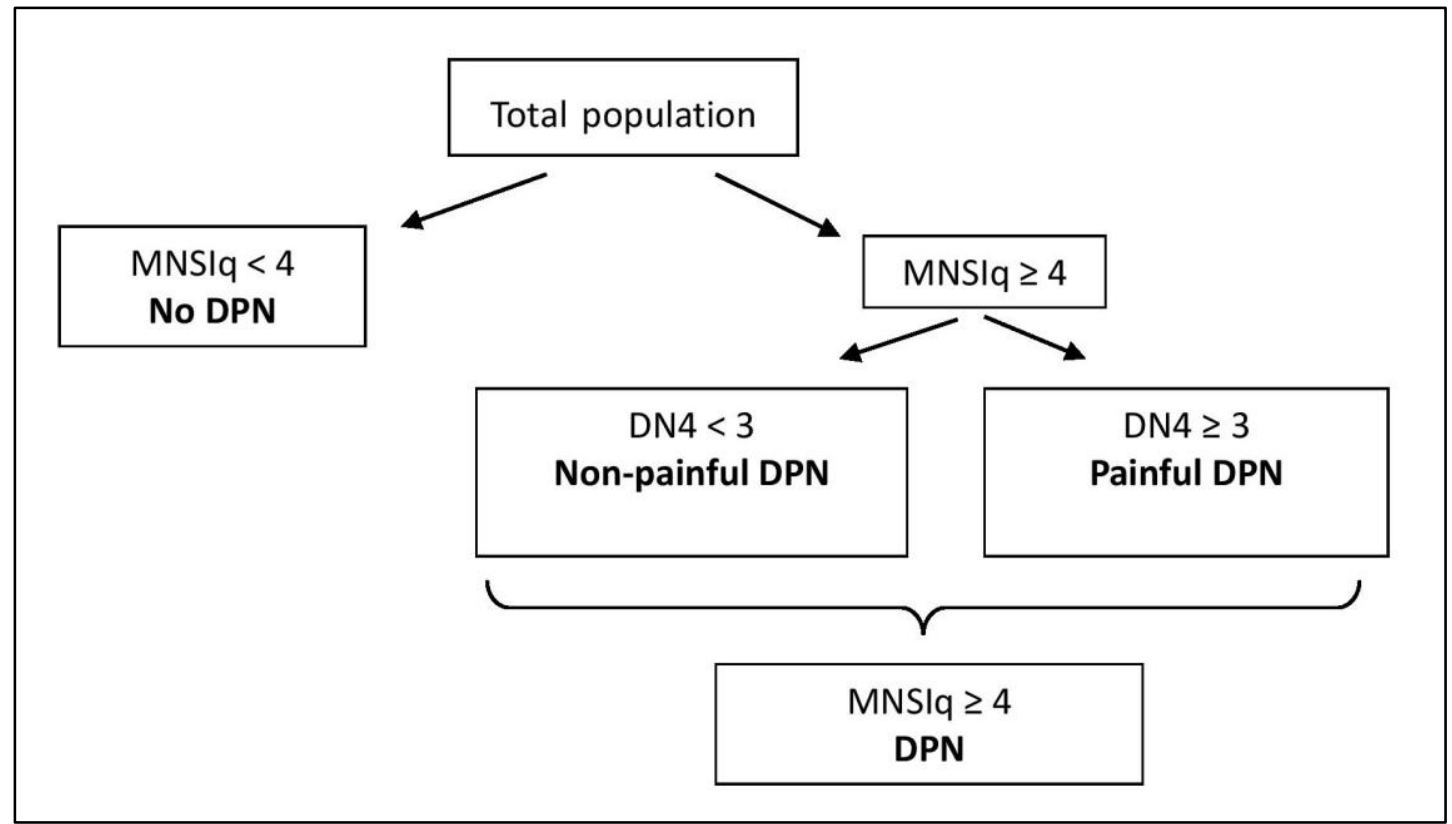

B)

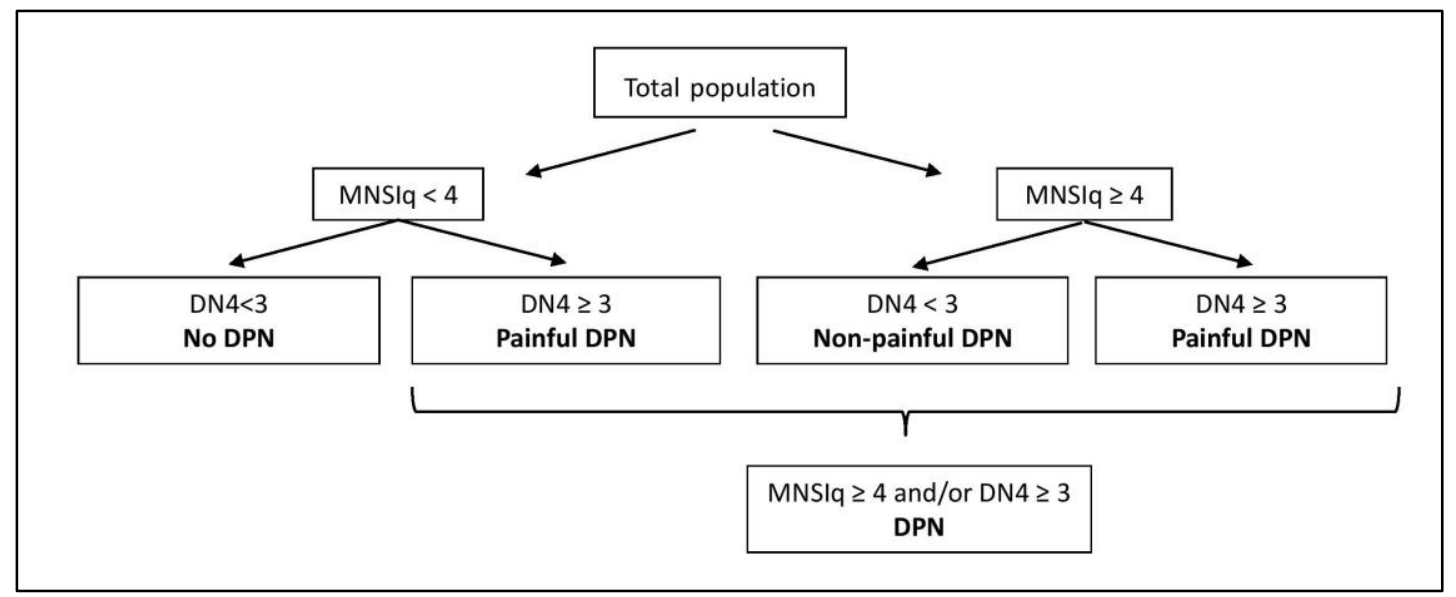

Of note, the DN4 score related specifically to pain in both feet and the DN4 questionnaire was only to be filled if pain in the feet was present. Thus, DN4 $<3$ means either no pain in feet or pain in both feet but DN4 $<3$. 
Supplementary Figure 3: Restricted cubic spline regression of DPN for central and general obesity measures.

Panel A: BMI, 20 years

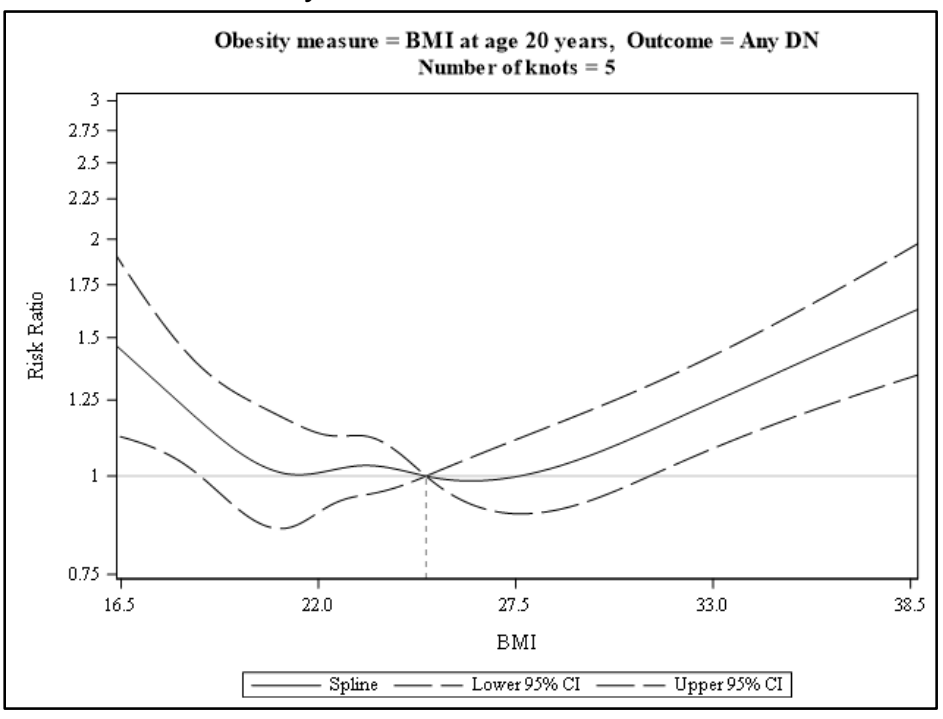

Panel C: BMI, questionnaire date

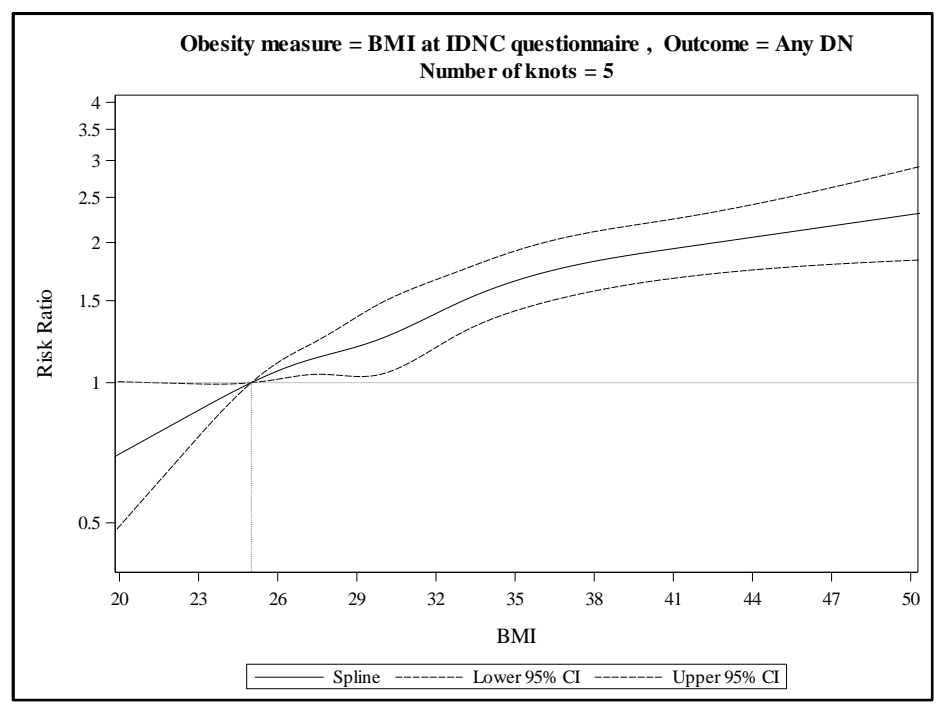

Panel B: BMI, baseline

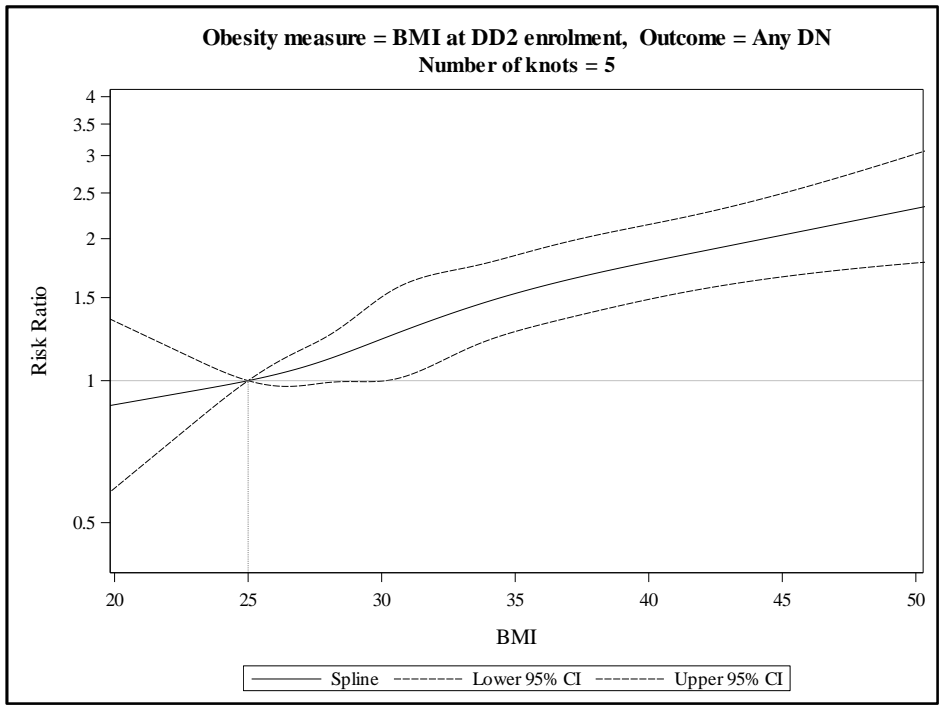

Panel D: BMI change (baseline -> questionnaire)

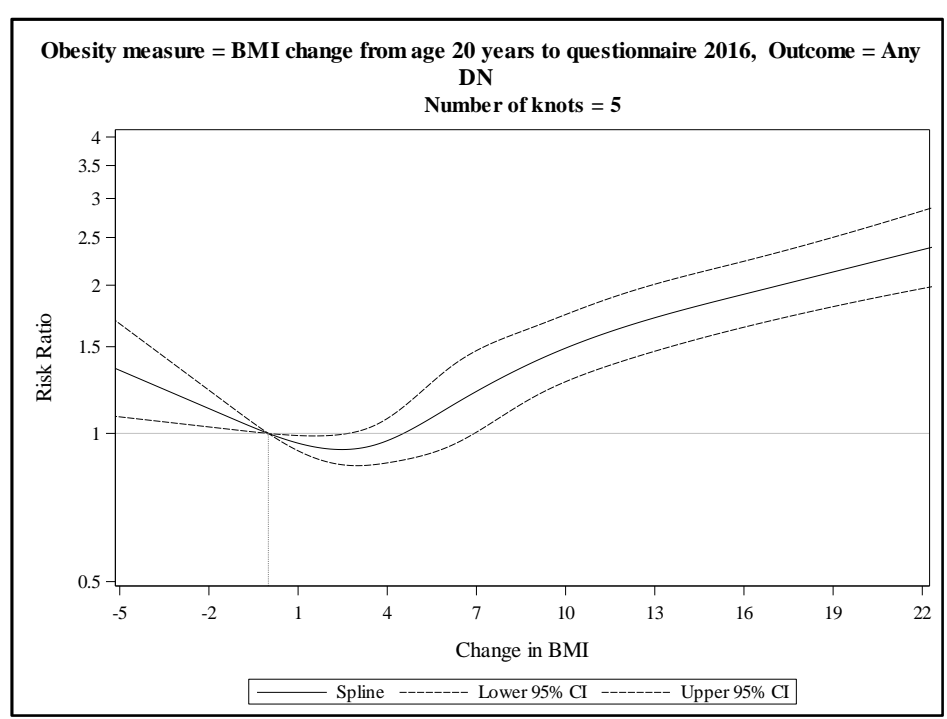


Panel E: Waist circumference, female

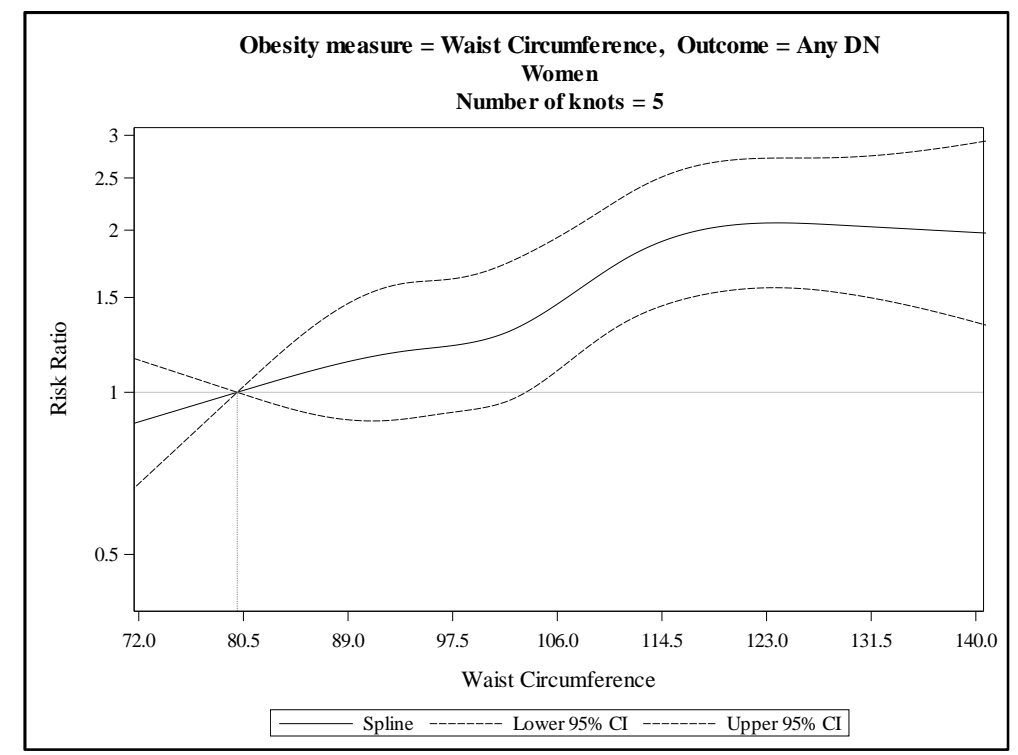

Panel G: Waist-hip ratio, female

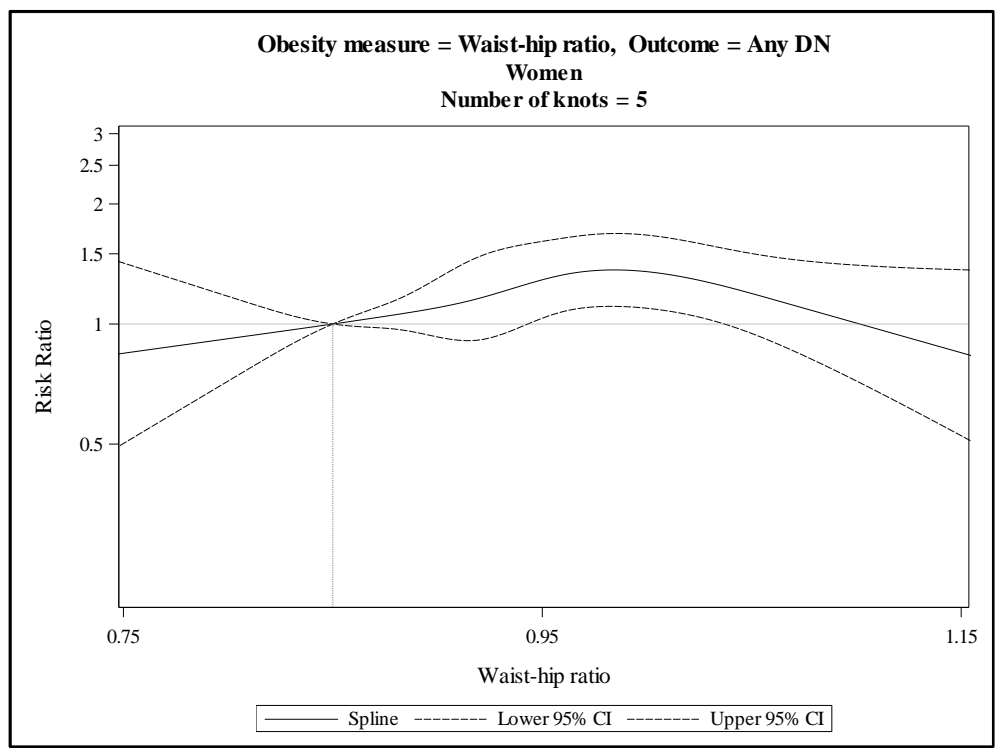

Panel F: Waist circumference, male

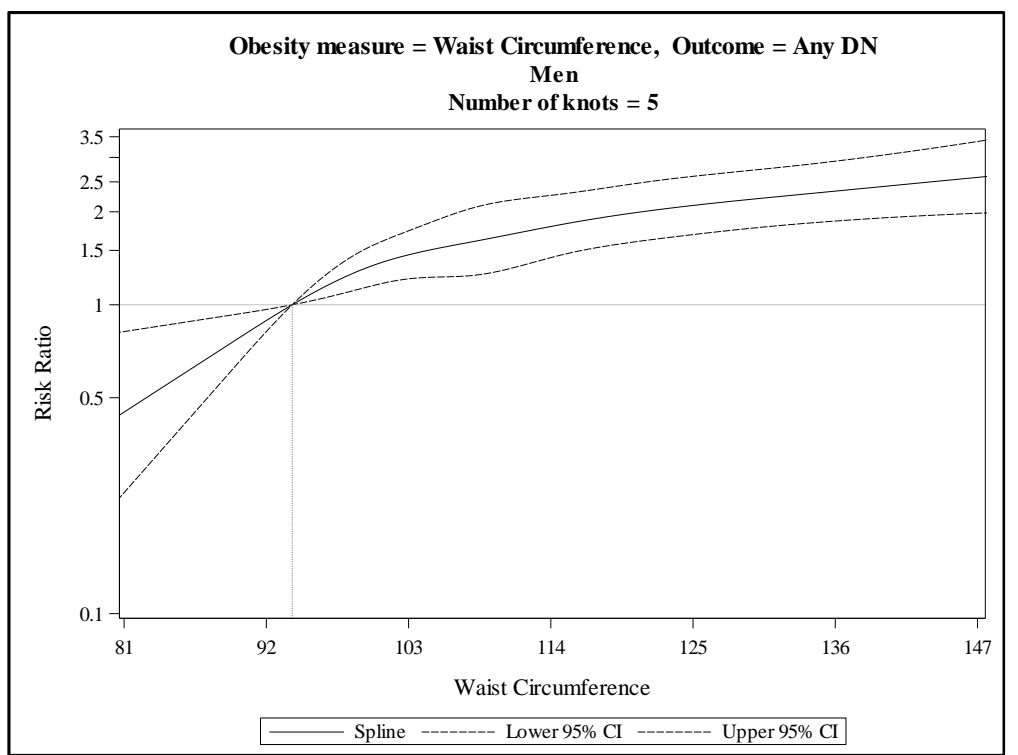

Panel H: waist-hip ratio, male

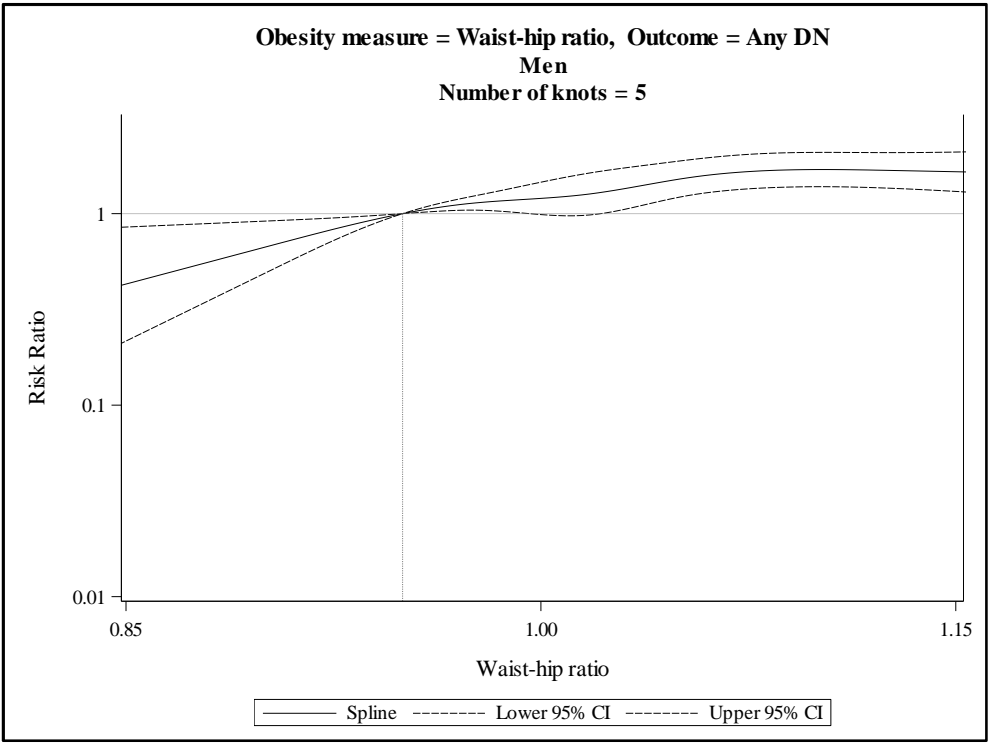


Panel I: waist-heigth ratio, female

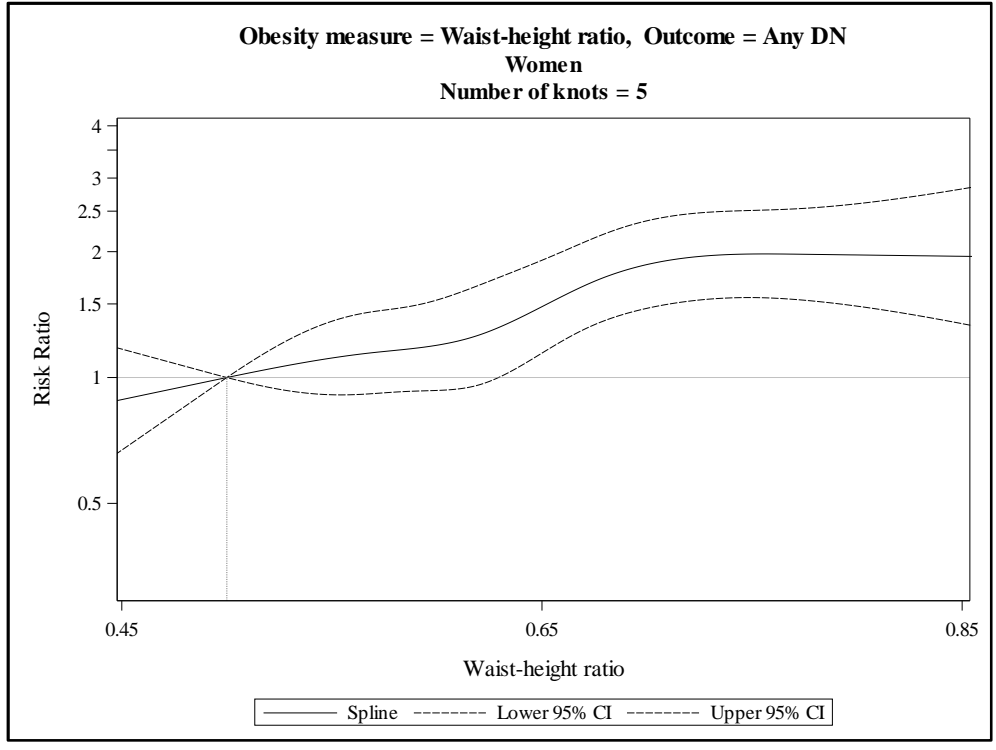

Panel J: Waist-heigth ratio ratio, male

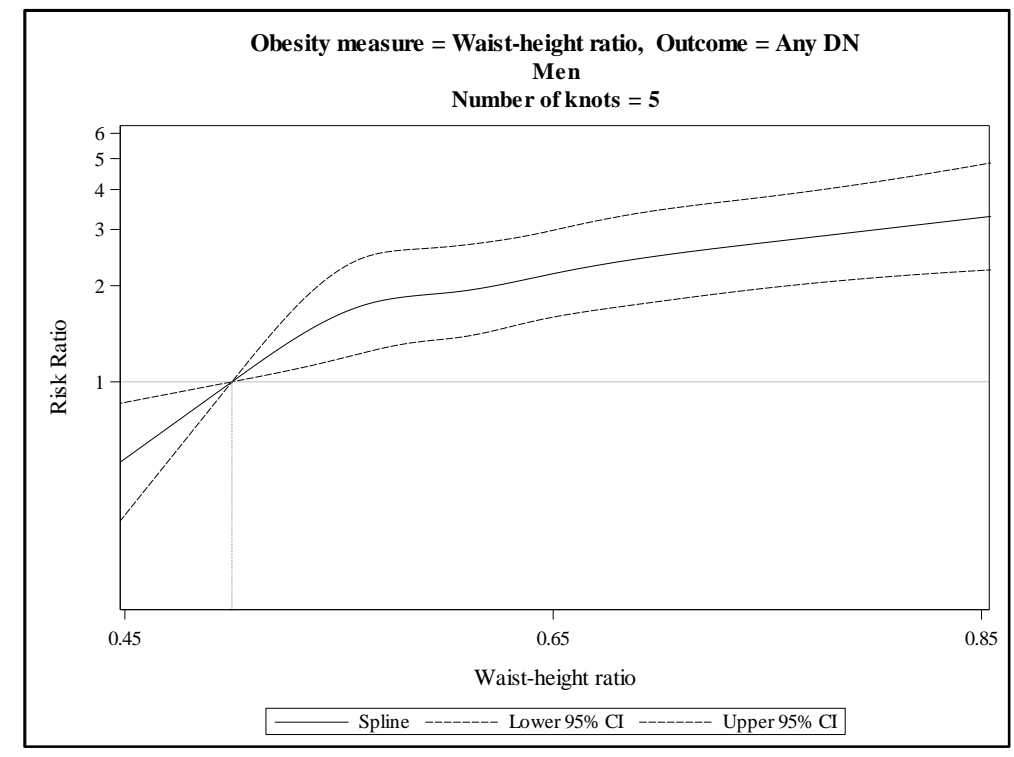


Supplementary Figure 4: Prevalence ratios of DPN for different obesity measures, without and with additional adjustment for BMI.

\begin{tabular}{|c|c|c|c|c|}
\hline & Total & Events & DPN & aPR $(95 \% \mathrm{Cl})$ \\
\hline Waist circumference (M/F) & 5239 & & I & \\
\hline$<94 / 80$ & 438 & 34 & - & 1.00 \\
\hline $94-101 / 80-87$ & 837 & 109 & $\mid \longmapsto-1$ & $1.67(1.16-2.41)$ \\
\hline 94-101/80-87, BMl-adjusted & & & $\mapsto-1$ & $1.52(1.05-2.20)$ \\
\hline$\geq 102 / 88$ & 3964 & 794 & $\longmapsto-1$ & $2.45(1.78-3.40)$ \\
\hline ¿102/88, BMl-adjusted & & & $\mapsto-1$ & $1.86(1.32-2.61)$ \\
\hline Waist-hip ratio (M/F) & 5237 & & 1 & \\
\hline$<0.95 / 0.85$ & 813 & 99 & $\theta$ & 1.00 \\
\hline $0.95-0.99 / 0.85-0.89$ & 1163 & 182 & $1 \bullet-1$ & $1.27(1.01-1.59)$ \\
\hline $0.95-0.99 / 0.85-0.89$, BMI-adjusted & & & $1 \cdot-1$ & $1.20(0.96-1.51)$ \\
\hline $1.00-1.04 / 0.90-0.94$ & 1629 & 311 & $1 *-1$ & $1.55(1.28-1.91)$ \\
\hline $1.00-1.04 / 0.90-0.94$, BMI-adjusted & & & $1 \mapsto \bullet-1$ & $1.41(1.14-1.74)$ \\
\hline$\geq 1.05 / 0.95$ & 1632 & 345 & $1 \bullet-1$ & $1.67(1.36-2.05)$ \\
\hline $21.05 / 0.95$, BMl-adjusted & & & $\mid \mapsto \bullet-1$ & $1.44(1.17-1.78)$ \\
\hline Waist-height ratio & 5210 & & | & \\
\hline$<0.50$ & 287 & 28 & $\phi$ & 1.00 \\
\hline $0.50-0.60$ & 1991 & 277 & $\mapsto$ & $1.51(1.04-2.17)$ \\
\hline $0.50-0.60$, BMI-adjusted & & & $H \bullet-1$ & $1.32(0.91-1.92)$ \\
\hline 20.60 & 2932 & 624 & $\longmapsto$ & $2.22(1.55-3.17)$ \\
\hline$\supseteq 0.60$, BMl-adjusted & & & $1 \longmapsto$ & $1.62(1.11-2.37)$ \\
\hline
\end{tabular}

Abbreviations: aPR; adjusted prevalence ratio, DPN; diabetic polyneuropathy, Cl; confidence interval; BMI; body mass index.

Of note: BMI from questionnaire 2016 is used for adjustment because this BMI measure is available for $\mathrm{N}=5159$, whereas $\mathrm{BMI}$ at baseline = DD2 enrollment is available only for $\mathrm{N}=3263$. Only a minor difference in BMI was observed between these two time points (median change: $0.4 \mathrm{~kg} / \mathrm{m}$ [IQR: -1.6 ; 0.6]) and central obesity measures were also associated with DPN independent of BMI if adjusted for BMI at baseline instead of BMI at questionnaire. All estimates are adjusted for age, sex, and diabetes duration. 